\title{
Moment angle complexes and big Cohen-Macaulayness
}

\author{
SHISEN LUO \\ TOMOO MATSUMURA \\ W FRANK MOORE
}

Let $\mathcal{Z}_{K} \subset \mathbb{C}^{m}$ be the moment angle complex associated to a simplicial complex $K$ on $[m]$, together with the natural action of the torus $\mathrm{T}=\mathrm{U}(1)^{m}$. Let $\mathrm{G} \subset \mathrm{T}$ be a (possibly disconnected) closed subgroup and $\mathrm{R}:=\mathrm{T} / \mathrm{G}$. Let $\mathbb{Z}[K]$ be the StanleyReisner ring of $K$ and consider $\mathbb{Z}\left[\mathrm{R}^{*}\right]:=H^{*}(B \mathrm{R} ; \mathbb{Z})$ as a subring of $\mathbb{Z}\left[\mathrm{T}^{*}\right]:=$ $H^{*}(B \mathrm{~T} ; \mathbb{Z})$. We prove that $H_{\mathrm{G}}^{*}\left(\mathcal{Z}_{K} ; \mathbb{Z}\right)$ is isomorphic to $\operatorname{Tor}_{\mathbb{Z}\left[\mathrm{R}^{*}\right]}^{*}(\mathbb{Z}[K], \mathbb{Z})$ as a graded module over $\mathbb{Z}\left[\mathrm{T}^{*}\right]$. Based on this, we characterize the surjectivity of $H_{\top}^{*}\left(\mathcal{Z}_{K} ; \mathbb{Z}\right) \rightarrow H_{\mathrm{G}}^{*}\left(\mathcal{Z}_{K} ; \mathbb{Z}\right.$ ) (ie $H_{\mathrm{G}}^{\text {odd }}\left(\mathcal{Z}_{K} ; \mathbb{Z}\right)=0$ ) in terms of the vanishing of $\operatorname{Tor}_{1}^{\mathbb{Z}\left[\mathrm{R}^{*}\right]}(\mathbb{Z}[K], \mathbb{Z})$ and discuss its relation to the freeness and the torsion-freeness of $\mathbb{Z}[K]$ over $\mathbb{Z}\left[\mathrm{R}^{*}\right]$. For various toric orbifolds $\mathcal{X}$, by which we mean quasitoric orbifolds or toric Deligne-Mumford stacks, the cohomology of $\mathcal{X}$ can be identified with $H_{\mathrm{G}}^{*}\left(\mathcal{Z}_{K}\right)$ with appropriate $K$ and $\mathrm{G}$ and the above results mean that $H^{*}(\mathcal{X} ; \mathbb{Z}) \cong \operatorname{Tor}_{\mathbb{Z}\left[\mathrm{R}^{*}\right]}^{*}(\mathbb{Z}[K], \mathbb{Z})$ and that $H^{\text {odd }}(\mathcal{X} ; \mathbb{Z})=0$ if and only if $H^{*}(\mathcal{X} ; \mathbb{Z})$ is the quotient $H_{\mathrm{R}}^{*}(\mathcal{X} ; \mathbb{Z})$.

55N91; 57R18, 53D20, 14M25

\section{Introduction}

The equivariant cohomology and the ordinary cohomology with $\mathbb{Z}$-coefficients of a "compact smooth toric space" (including quasitoric manifolds, complete smooth toric varieties) is known by the work of Danilov [8], Jurkiewicz [19] and Davis and Januszkiewicz [9]: the equivariant cohomology is the Stanley-Reisner ring of the associated simplicial complex and the ordinary cohomology is the quotient of the equivariant cohomology by linear relations.

The orbifold analogue of these spaces has also been introduced and studied by several people, for example, Lerman and Tolman [21], Borisov, Chen and Smith [3] and Poddar and Sarkar [25]. The equivariant cohomology of these toric orbifolds with $\mathbb{Z}-$ coefficients is also known to be the associated Stanley-Reisner rings and the ordinary cohomology is the quotient of the equivariant cohomology over $\mathbb{Q}$-coefficients; cf [3; 
$8 ; 19 ; 25]$. However the ordinary cohomology with $\mathbb{Z}$-coefficients is hard to compute because it is not the quotient of the equivariant cohomology in general (for example in the case of a direct product of weighted projective spaces). The main theme of this paper is to characterize when the ordinary cohomology is the quotient of the equivariant cohomology.

Our approach is to view previously mentioned toric orbifolds as quotient stacks given by partial quotients of moment angle complexes and consider the cohomology of stacks in the sense of Edidin [10] (see also Tolman [28] and Edidin and Graham [11]). The moment-angle complex $\mathcal{Z}_{K}$ was introduced by Buchstaber and Panov in [6] as a disc-circle decomposition of the Davis-Januszkiewicz universal space associated to a simplicial complex $K$ [9] where they introduced a quasitoric manifold as a partial quotient of the moment angle manifold associated to a simple polytope.

If $K$ is a simplicial complex on $[m]:=\{1, \ldots, m\}$, then $\mathcal{Z}_{K}$ carries a natural action of the torus $\mathrm{T}:=\mathrm{U}(1)^{m}$. The quotient stack $\left[\mathcal{Z}_{K} / \mathrm{G}\right]$ with an appropriate choice of the subgroup $\mathrm{G} \subset \mathrm{T}$ can be used as a topological model to compute the cohomology of quasitoric orbifolds [9; 25], symplectic toric orbifolds [21] and toric Deligne-Mumford stacks [3]. In other words, the ordinary cohomology of these toric orbifolds as stacks can be defined as the $\mathrm{G}$-equivariant cohomology $H_{\mathrm{G}}^{*}\left(\mathcal{Z}_{K} ; \mathbb{Z}\right)$. Similarly the equivariant cohomology can be defined as $H_{\mathrm{T}}^{*}\left(\mathcal{Z}_{K} ; \mathbb{Z}\right)$ which is isomorphic to the Stanley-Reisner $\operatorname{ring} \mathbb{Z}[K]$ as quotient rings of $\mathbb{Z}\left[T^{*}\right]:=H^{*}(B T ; \mathbb{Z})=\mathbb{Z}\left[x_{1}, \ldots, x_{m}\right]$. In Section 2 , we recall the constructions of those toric orbifolds and the relation to moment angle complexes to motivate our readers.

In Section 3, we start with proving the following.

Theorem A Let $\mathrm{G} \subset \mathrm{T}$ be a (possibly disconnected) closed subgroup and $\mathrm{R}:=\mathrm{T} / \mathrm{G}$. There is an isomorphism of graded $\mathbb{Z}$-modules:

$$
H_{\mathrm{G}}^{*}\left(\mathcal{Z}_{K} ; \mathbb{Z}\right) \cong \operatorname{Tor}_{\mathbb{Z}\left[\mathrm{R}^{*}\right]}^{*}(\mathbb{Z}[K] ; \mathbb{Z})
$$

Furthermore there is a natural commutative diagram:

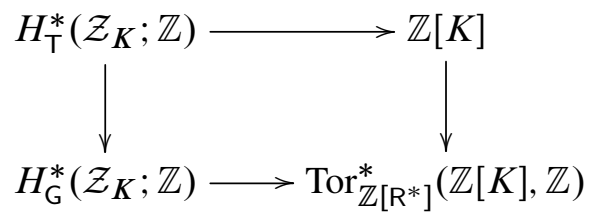

Here $\mathbb{Z}\left[\mathrm{R}^{*}\right]:=H^{*}(B \mathrm{R} ; \mathbb{Z})=\mathbb{Z}\left[u_{1}, \ldots, u_{n}\right]$ is considered as a subring of $\mathbb{Z}\left[\mathrm{T}^{*}\right]$ so that the $u_{i}$ are linear combinations of the $x_{j}$. It is worth noting that this theorem holds more generally. Namely, the theorem holds for any topological space $X$ with T-action 
such that $C^{*}\left(E \mathrm{~T} \times_{\mathrm{T}} X ; \mathbb{Z}\right)$ is formal in the category of $H^{*}(B \mathrm{~T} ; \mathbb{Z})$-modules up to homotopy in the sense of Franz [13]. See Section 3 for the details.

Based on this isomorphism, we prove our characterization theorem.

Theorem B The following conditions are equivalent:

(i) $\operatorname{Tor}_{1}^{\mathbb{Z}\left[\mathrm{R}^{*}\right]}(\mathbb{Z}[K], \mathbb{Z})=0$

(ii) $H_{\mathrm{G}}^{*}\left(\mathcal{Z}_{K} ; \mathbb{Z}\right)$ is isomorphic to the quotient of $\mathbb{Z}[K]$ by linear forms

(iii) $H_{\mathrm{G}}^{\text {odd }}\left(\mathcal{Z}_{K} ; \mathbb{Z}\right)=0$

We will explain in Section 4 that, even though $\mathbb{Z}[K]$ might not be finitely generated over $\mathbb{Z}\left[\mathrm{R}^{*}\right]$, the vanishing of $\operatorname{Tor}_{1}^{\mathbb{Z}\left[\mathrm{R}^{*}\right]}(\mathbb{Z}[K], \mathbb{Z})$ has the usual meaning in terms of regular sequences, ie $\operatorname{Tor}_{1}^{\mathbb{Z}\left[\mathrm{R}^{*}\right]}(\mathbb{Z}[K], \mathbb{Z})=0$ if and only if $u_{1}, \ldots, u_{n}$ form a $\mathbb{Z}[K]$-regular sequence. Thus we say $\mathbb{Z}[K]$ is big Cohen-Macaulay over $\mathbb{Z}\left[\mathrm{R}^{*}\right]$ if (i) is satisfied.

By presenting a toric orbifold $\mathcal{X}$ as $\left[\mathcal{Z}_{K} / G\right]$, we obtain the following immediate corollary.

Corollary $\mathbf{C}$ If $\mathcal{X}$ is a toric orbifold stack presented as $\left[\mathcal{Z}_{K} / G\right]$, then

$$
H^{*}(\mathcal{X} ; \mathbb{Z}) \cong \operatorname{Tor}_{\mathbb{Z}\left[R^{*}\right]}^{*}(\mathbb{Z}[K] ; \mathbb{Z}) .
$$

Also, $H^{*}(\mathcal{X} ; \mathbb{Z})$ is the quotient of Stanley-Reisner ring $\mathbb{Z}[K]$ if and only if one of the following equivalent conditions holds: (i) $H^{\text {odd }}(\mathcal{X} ; \mathbb{Z})=0$; (ii) $\operatorname{Tor}_{1}^{\mathbb{Z}\left[\mathrm{R}^{*}\right]}(\mathbb{Z}[K] ; \mathbb{Z})=0$.

For example, the cohomology of weighted projective spaces as stacks are shown to be the quotient of its equivariant cohomology, based on the computation exhibited in Holm [17]. On the other hand, the cohomology of a direct product of weighted projective spaces is not the quotient of its equivariant cohomology. See Section 6 for the details and more examples.

In Section 5, we will discuss the freeness and the torsion-freeness of $\mathbb{Z}[K]$ over $\mathbb{Z}\left[\mathrm{R}^{*}\right]$. In particular, we show that the equivariant cohomology of a toric orbifold is torsionfree over $\mathbb{Z}\left[\mathrm{R}^{*}\right]$. We also give an injectivity theorem on the equivariant cohomology of $\mathcal{Z}_{K}$ (Theorem 5.14) for a symplectic toric orbifold $\left[\mathcal{Z}_{K} / \mathrm{G}\right]$, which gives a sufficient condition that $\mathbb{Z}[K]$ is free over a subring of $\mathbb{Z}\left[\mathrm{R}^{*}\right]$.

Finally, in Section 7, in light of Theorem 3.3, we construct an algebraic Gysin sequence for Tor of $\mathbb{Z}[K]$ in analogy with the Gysin sequence of $S^{1}$-fibration over a toric manifold. 
Acknowledgements The authors want to thank M Franz, T Holm, Y Karshon, A Knutson, T Ohmoto, K Ono and D Suh for important advice and useful conversations, as well as the referee for a thorough reading that improved the paper greatly. The first author is particularly indebted to K Ono for providing him an excellent environment at Hokkaido University where he had spent significant time for this paper in July and August 2011. The first author would like to show his gratitude to the Algebraic Structure and its Application Research Center (ASARC) at KAIST for its constant support. The first author is also supported by the National Research Foundation of Korea (NRF) grant funded by the Korea government (MEST) (number 2012-0000795, 2011-0001181).

\section{Moment angle complexes and toric orbifolds}

In this section, we review the basic facts about moment angle complexes and various toric orbifolds to motivate our results.

\subsection{Moment angle complexes}

The moment angle complex $\mathcal{Z}_{K}$ associated to a simplicial complex $K$ was introduced by Buchstaber and Panov in [6] as a disc-circle decomposition of the Davis-Januszkiewicz universal space associated to a simplicial complex $K$ [9] and it has been actively studied in toric topology and its connections to symplectic and algebraic geometry and combinatorics. For convenience, we use the following notation for the rest of the paper.

Notation 2.1 Let $X, Y$ be subsets of a set $Z$. For a subset $\sigma \subset[m], X^{\sigma} \times Y^{[m] \backslash \sigma} \subset Z^{m}$ is the direct product of $X$ 's and $Y$ 's where $i^{\text {th }}$ component is $X$ if $i \in \sigma$ and $Y$ if $i \notin \sigma$.

For a simplicial complex $K$ on vertices $[m]:=\{1, \ldots, m\}$, the moment angle complex $\mathcal{Z}_{K} \subset \mathbb{C}^{m}$ is defined as $\mathcal{Z}_{K}=\bigcup_{\sigma \in K} \mathrm{D}^{\sigma} \times(\partial \mathrm{D})^{[m] \backslash \sigma}$, where $\mathrm{D}=\{z \in \mathbb{C}|| z \mid \leq 1\}$ is the unit disk and $\partial \mathrm{D}$ is its boundary circle. This space $\mathcal{Z}_{K}$ carries a natural action of $\mathrm{T}=\mathrm{U}(1)^{m}$. It is originally proved in [9] that

$$
H_{\mathrm{T}}^{*}\left(\mathcal{Z}_{K} ; \mathbb{Z}\right) \cong \mathbb{Z}[K] \text { as graded } \mathbb{Z}\left[\mathrm{T}^{*}\right] \text {-algebras. }
$$

Here, $\mathbb{Z}[K]=\mathbb{Z}\left[x_{1}, \ldots, x_{m}\right] /\left\langle x_{\sigma}, \sigma \notin K\right\rangle$ is the Stanley-Reisner (face) ring, where $x_{\sigma}:=\prod_{i \in \sigma} x_{i}$. With the identification $\mathbb{Z}\left[\mathrm{T}^{*}\right]:=H^{*}(B \mathrm{~T} ; \mathbb{Z})=\mathbb{Z}\left[x_{1}, \ldots, x_{m}\right]$, the isomorphism is as graded algebras over the polynomial ring with $\operatorname{deg} x_{i}=2$. For the details, we refer to Buchtaber and Panov [5, Chapter 6].

Baskakov, Buchstaber and Panov [2] also computed the ordinary cohomology of $\mathcal{Z}_{K}$,

$$
H^{*}\left(\mathcal{Z}_{K} ; \mathbb{Z}\right) \cong \operatorname{Tor}_{\mathbb{Z}\left[T^{*}\right]}^{*}(\mathbb{Z}[K], \mathbb{Z}) \text { as graded rings. }
$$


Here the grading on the right hand side is the total degree of bidegree coming from the (co)homological degree of Koszul complex and the degree of $\mathbb{Z}[K]$, as is explained in the following definition.

Definition 2.2 Let $M$ be a graded $\mathbb{Z}\left[\mathrm{R}^{*}\right]:=\mathbb{Z}\left[u_{1}, \ldots, u_{n}\right]$-module. Let $\Lambda$ be the exterior algebra generated by $\eta_{1}, \ldots, \eta_{n}$ with $\operatorname{deg} \eta_{i}=1$. Let $\mathrm{M} \otimes^{\mathrm{R}} \Lambda$ be the corresponding Koszul complex. Its homology is $\operatorname{Tor}_{*}^{\mathbb{Z}\left[R^{*}\right]}(M, \mathbb{Z})$ where the degree is given by $\operatorname{deg} \eta_{i}=1$. The complex $\mathrm{M} \otimes^{\mathrm{R}} \Lambda$ is also a bigraded differential complex with $\operatorname{bideg}\left(\alpha \otimes \xi_{i}\right)=[-1,2]+[0,|\alpha|]$, where $\alpha \in M^{|\alpha|}$. The cohomological degree of Tor is defined to be the total degree of this bidegree and is denoted by the superscript as in $\operatorname{Tor}_{\mathbb{Z}\left[R^{*}\right]}^{*}(M, \mathbb{Z})$.

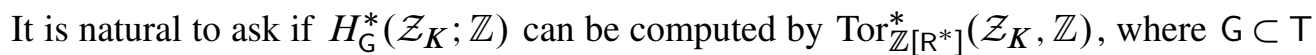
is a (possibly disconnected) closed subgroup, $\mathrm{R}:=\mathrm{T} / \mathrm{G}$ and $\mathbb{Z}\left[\mathrm{R}^{*}\right]:=H^{*}(B \mathrm{R} ; \mathbb{Z}) \subset$ $\mathbb{Z}\left[T^{*}\right]$. In Section 3, we show that

$$
H_{\mathrm{G}}^{*}\left(\mathcal{Z}_{K} ; \mathbb{Z}\right) \cong \operatorname{Tor}_{\mathbb{Z}\left[\mathrm{R}^{*}\right]}^{*}\left(\mathcal{Z}_{K}, \mathbb{Z}\right) \text { as graded } \mathbb{Z}\left[\mathrm{T}^{*}\right] \text {-modules (Theorem 3.3). }
$$

\subsection{Partial quotient of moment angle complexes}

Various toric "spaces," such as quasitoric orbifolds defined by Poddar and Sarkar [25], symplectic compact toric orbifolds defined by Lerman and Tolman [21] and algebraic toric orbifolds defined by Borisov, Chen and Smith [3], can be topologically regarded as the quotient stack $\left[\mathcal{Z}_{K} / G\right]$ with an appropriate choice of subgroup $\mathrm{G} \subset \mathrm{T}$ that acts on $\mathcal{Z}_{K}$ locally freely. In the next section, we recall the construction of those spaces and see that the cohomology rings of all of these toric spaces in nice cases are computed as the quotient of the Stanley-Reisner ring $\mathbb{Z}[K]$. In this section, we just give a criteria for the local freeness of the action of a closed subgroup $\mathrm{G}$ of $\mathrm{T}$ on $\mathcal{Z}_{K}$ and a remark about the cohomology of orbifolds as stacks.

Lemma 2.3 Let $n$ be the largest cardinality of a face in a pure simplicial complex $K$. If a closed subgroup $\mathrm{G} \subset \mathrm{T}$ acts on $\mathcal{Z}_{K}$ locally freely, then $\operatorname{dim} \mathrm{G} \leq m-n$. Furthermore, if $n$ is the cardinality of maximal faces in $K$ and $\operatorname{dim} \mathrm{G}=m-n$, then $\mathrm{G}$ acts on $\mathcal{Z}_{K}$ locally freely if and only if $\mathrm{T}_{\sigma}:=\mathrm{U}(1)^{\sigma} \times\{1\}^{[m] \backslash \sigma} \subset \mathrm{T}=\mathrm{U}(1)^{m}$ surjects to $\mathrm{R}:=\mathrm{T} / \mathrm{G}$ for all maximal faces $\sigma$.

Proof Let $\sigma \in K$ such that $|\sigma|=n$. Let $0_{\sigma} \in \mathrm{D}^{\sigma} \times(\partial \mathrm{D})^{[m] \backslash \sigma}$ such that $i^{\text {th }}$ component of $0_{\sigma}$ for $i \in \sigma$ is $0 \in \mathrm{D}$. Then the stabilizer of $0_{\sigma}$ in $\mathrm{T}$ is $T_{\sigma}:=\mathrm{U}(1)^{\sigma} \times\{1\}^{[m] \backslash \sigma} \subset$ $\mathrm{T}=\mathrm{U}(1)^{m}$. Consider the map $\theta: \mathrm{G} \times \mathrm{T}_{\sigma} \rightarrow \mathrm{T},(g, t) \mapsto g t$. The kernel of this map $\theta$ is finite if and only if the stabilizer of $0_{\sigma}$ in $\mathrm{G}$ is finite. Therefore the local freeness of 
the $\mathrm{G}$-action implies that the dimension of the image of $\theta$ is $\operatorname{dim} \mathrm{G}+|\sigma|=\operatorname{dim} \mathrm{G}+n$. Thus $\operatorname{dim} \mathrm{G} \leq m-n$ since $\operatorname{dim} \mathrm{T}=m$. To prove the latter claim, note that the local freeness of the $\mathrm{G}$-action is equivalent to the stabilizer of $0_{\sigma}$ in $\mathrm{G}$ being finite for each maximal face $\sigma$. Since $\mathrm{G}=m-n$ and $\operatorname{dim} \mathrm{T}_{\sigma}, \mathrm{G} \cap \mathrm{T}_{\sigma}$ is zero-dimensional if and only if $\mathrm{T}_{\sigma} \rightarrow \mathrm{R}$ is surjective.

Remark 2.4 In [10], Edidin defined the integral cohomology of a stack and showed that if the stack is given as a global quotient stack $[M / \mathrm{G}]$, then $H^{*}([M / \mathrm{G}] ; \mathbb{Z})$ is canonically isomorphic to $H_{\mathrm{G}}^{*}(M ; \mathbb{Z})$. Therefore when $[M / \mathrm{G}]$ is an orbifold, the cohomology of the orbifold $[M / \mathrm{G}]$ as a stack is also $H_{\mathrm{G}}^{*}(M ; \mathbb{Z})$. On the other hand, the projection map $B \mathrm{G} \times{ }_{\mathrm{G}} M \rightarrow M / \mathrm{G}$, where $M / \mathrm{G}$ is the quotient topological space, induces an isomorphism $H_{\mathrm{G}}^{*}(M ; \mathbb{Q}) \cong H^{*}(M / \mathrm{G} ; \mathbb{Q})$ since the fiber is " $\mathbb{Q}$-acyclic". If $\mathrm{G}$ acts freely on $M$, then $H^{*}([M / \mathrm{G}] ; \mathbb{Z}) \cong H_{\mathrm{G}}^{*}(M ; \mathbb{Z}) \cong H^{*}(M / \mathrm{G} ; \mathbb{Z})$. If $\mathrm{L}$ acts on $M$ and $\mathrm{G}$ is a subgroup of $\mathrm{L}$ that acts on $M$ locally freely, we have the action of $\mathrm{K}:=\mathrm{L} / \mathrm{G}$ on the orbifold $[M / \mathrm{G}]$. In this case, there is an isomorphism of stacks $[[M / \mathrm{G}] / \mathrm{K}] \cong[M / \mathrm{L}]$ and we can define $H_{\mathrm{K}}^{*}([M / \mathrm{G}] ; \mathbb{Z}):=H^{*}([M / \mathrm{L}] ; \mathbb{Z})=$ $H_{\mathrm{L}}^{*}(M ; \mathbb{Z})$; cf Romagny [26] and Lerman and Malkin [20].

Remark 2.5 The following is a useful criteria for the connectedness of $\mathrm{G}$. Let $B$ be the integer matrix induced from the quotient map $T \rightarrow R$. Then $G$ is connected if and only if $B: \mathbb{Z}^{m} \rightarrow \mathbb{Z}^{n}$ is surjective.

\subsection{Quasitoric orbifolds}

Quasitoric manifolds are introduced and studied by Davis and Januszkiewicz [9] and its orbifold counterpart is studied by Poddar and Sarkar [25]. Let $\Delta$ be a simple polytope of dimensional $n$ in $\mathrm{R}^{n}$. Let $H_{1}, \ldots, H_{m}$ be the facets of $\Delta$ and for a face $F_{\sigma}=\bigcap_{i \in \sigma} H_{i}$, let $\mathrm{T}_{\sigma}:=\mathrm{U}(1)^{\sigma} \times\{1\}^{[m] \backslash \sigma} \subset \mathrm{T}=\mathrm{U}(1)^{m}$. Define $\mathcal{Z}_{\Delta}:=\mathrm{T} \times \Delta / \sim$, where $\left(t_{1}, p\right) \sim\left(t_{2}, q\right)$ if and only if $p=q$ is contained in a relative interior of $F_{\sigma}$ and $t_{1} t_{2}^{-1} \in \mathrm{T}_{\sigma}$; $\operatorname{cf}\left[5\right.$, Definition 6.1]. It is known that $\mathcal{Z}_{\Delta}$ is a smooth manifold; cf [5, Lemma 6.2]. Let $B$ be an integer $n \times m$ matrix such that for each vertex $H_{i_{1}} \cap \cdots \cap H_{i_{n}}$ of $\Delta$, the corresponding columns $\lambda_{i_{1}}, \ldots, \lambda_{i_{n}}$ form a basis of $\mathbb{Q}^{n}$. By the assumption, $B$ defines a surjective map $\mathrm{T} \rightarrow \mathrm{R}$ also denoted by $B$. Let $\mathrm{G}$ be the kernel of $B$. A quasitoric orbifold for the pair $(\Delta, B)$ is defined as the quotient stack $\left[\mathcal{Z}_{\Delta} / \mathrm{G}\right]$. Here note that the assumption on $B$ is equivalent to the local freeness of the $\mathrm{G}-$ action on $\mathcal{Z}_{\Delta}$ (see Lemma 2.3). Since $\mathcal{Z}_{\Delta}$ is T-equivariantly homeomorphic to $\mathcal{Z}_{K_{\Delta}}$, where $K_{\Delta}$ is the simplicial complex associated to $\Delta$ (see [5, Section 6.2]), the quasitoric orbifold $\left[\mathcal{Z}_{\Delta} / \mathrm{G}\right]$ is topologically the quotient of the moment angle complex $\mathcal{Z}_{K_{\Delta}}$ by $\mathrm{G}$. 
As a consequence and by Remark 2.4, the R-equivariant cohomology of the quasitoric orbifold $\left[\mathcal{Z}_{\Delta} / \mathrm{G}\right]$ is

$$
H_{\mathrm{R}}^{*}\left(\left[\mathcal{Z}_{\Delta} / \mathrm{G}\right] ; \mathbb{Z}\right) \cong H_{\mathrm{T}}^{*}\left(\mathcal{Z}_{\Delta} ; \mathbb{Z}\right) \cong H_{\mathrm{T}}^{*}\left(\mathcal{Z}_{K_{\Delta}} ; \mathbb{Z}\right) \cong \mathbb{Z}\left[K_{\Delta}\right]
$$

The rational cohomology ring of the quasitoric orbifold is computed by Poddar and Sarkar [25].

Theorem 2.6 (Poddar-Sarkar) If $\left[\mathcal{Z}_{\Delta} / \mathrm{G}\right]$ is a quasitoric orbifold,

$$
H^{*}\left(\left[\mathcal{Z}_{\Delta} / \mathrm{G}\right] ; \mathbb{Q}\right) \cong \mathbb{Q}\left[K_{\Delta}\right] /\left\langle u_{1}, \ldots, u_{n}\right\rangle \text {. }
$$

Here $u_{j}=\sum_{i=1}^{m} B_{j i} x_{i} \in \mathbb{Z}\left[x_{1}, \ldots, x_{m}\right]$ and we identify $H^{*}(B \mathrm{R} ; \mathbb{Z})=\mathbb{Z}\left[u_{1}, \ldots, u_{n}\right]$. A quasitoric orbifold given by $(\Delta, B)$ is a quasitoric manifold if and only if for each vertex $H_{i_{1}} \cap \cdots \cap H_{i_{n}}$ of $\Delta$, the corresponding columns $\lambda_{i_{1}}, \ldots, \lambda_{i_{n}}$ of $B$ form a $\mathbb{Z}$-basis. In this case, the isomorphism holds with $\mathbb{Z}$-coefficients.

Theorem 2.7 (Davis-Januszkiewicz [9]) If $\left[\mathcal{Z}_{\Delta} / \mathrm{G}\right]$ is a quasitoric manifold,

$$
H^{*}\left(\left[\mathcal{Z}_{\Delta} / \mathrm{G}\right] ; \mathbb{Z}\right) \cong \mathbb{Z}\left[K_{\Delta}\right] /\left\langle u_{1}, \ldots, u_{n}\right\rangle \text {. }
$$

Moreover $H^{*}\left(\left[\mathcal{Z}_{\Delta} / \mathrm{G}\right], \mathbb{Z}\right)$ has no $\mathbb{Z}$-torison (which follows from [9, Theorem 3.1] and the fact that a quasitoric manifold is closed and orientable).

Since $\mathcal{Z}_{\Delta}$ is T-equivariantly homeomorphic to $\mathcal{Z}_{K_{\Delta}}$, we have the following.

Corollary 2.8 If $K_{\Delta}$ and $B$ give a quasitoric orbifold, then

$$
H_{\mathrm{G}}^{*}\left(\mathcal{Z}_{K_{\Delta}} ; \mathbb{Q}\right) \cong \mathbb{Q}\left[K_{\Delta}\right] /\left\langle u_{1}, \ldots, u_{n}\right\rangle \text {. }
$$

If $K_{\Delta}$ and $B$ give a quasitoric manifold, then $H_{\mathrm{G}}^{*}\left(\mathcal{Z}_{K_{\Delta}} ; \mathbb{Z}\right) \cong \mathbb{Z}\left[K_{\Delta}\right] /\left\langle u_{1}, \ldots, u_{n}\right\rangle$.

\subsection{Compact symplectic toric orbifolds}

Lerman and Tolman [21] classified compact symplectic toric (effective) orbifolds in terms of labeled polytopes. A labeled polytope $(\Delta, \mathrm{b})$ is a rational simple polytope $\Delta$ in $\mathbb{R}^{n}$ with each facet $H_{i}, i=1, \ldots, m$ is labeled by a positive integer $b_{i}$. If $\beta_{i}$ is the integral primitive inward normal vector to each facet $H_{i}$, then by assigning the integer matrix $B=\left[\mathrm{b}_{1} \beta_{1}, \ldots, \mathrm{b}_{m} \beta_{m}\right]$, we obtain a quasitoric orbifold given by $(\Delta, B)$. Here the symplectic structure on $\left[\mathcal{Z}_{\Delta} / \mathrm{G}\right]$ comes from identifying $\mathcal{Z}_{\Delta}$ with the level set for the reduction of $\mathbb{C}^{m}$ by the action of G. A compact symplectic toric manifold is given by the labeled polytope such that $\mathrm{b}_{i}=1$, for all $i=1, \ldots, m$ and such that for each vertex $H_{i_{1}} \cap \cdots \cap H_{i_{n}}$ of $\Delta$, the corresponding primitive normal vectors $\beta_{i_{1}}, \ldots, \beta_{i_{n}}$ of $B$ form a $\mathbb{Z}$-basis. This is exactly the Delzant condition in the classification of compact symplectic manifolds. 


\subsection{Algebraic toric (effective) orbifold (also known as toric Deligne- Mumford stack)}

Let $K$ be a pure simplicial complex on $[m]$. Define a fan $\Sigma_{K}$ in $\mathbb{R}^{m}$ where each cone is generated by the part of standard basis $\mathrm{e}_{i}, i \in \sigma$ for each $\sigma \in K$. The corresponding toric variety $X_{\Sigma_{K}}$ is a smooth open subvariety of $\mathbb{C}^{m}$ that is exactly the complement of subspace arrangements given by $K$; $\operatorname{cf}$ [5, Chapter 8]. There is a natural embedding of $\mathcal{Z}_{K}$ into $X_{\Sigma_{K}}$.

Proposition 2.9 [5, Proposition 8.9] There is a T-equivariant deformation retract for $\mathcal{Z}_{K} \subset X_{\Sigma_{K}}$ and, in particular, $H_{\mathrm{G}}^{*}\left(\mathcal{Z}_{K} ; \mathbb{Z}\right) \cong H_{\mathrm{G}_{\mathbb{C}}}^{*}\left(X_{\Sigma_{K}} ; \mathbb{Z}\right)$ for each subgroup $\mathrm{G} \subset \mathrm{T}$ and its complexification $\mathrm{G}_{\mathbb{C}}$.

The algebraic toric orbifolds studied by Borisov, Chen and Smith [3] are defined by the stacky fan. There they consider possibly noneffective orbifolds. In this paper, since we are interested in the effective case, we simplify the stacky fan and call it the labeled fan. A labeled fan $(\Sigma, \mathrm{b})$ is a rational simplicial fan $\Sigma$ in $\mathbb{R}^{n}$ with each ray $\rho_{i}$ labeled by a positive integer $\mathrm{b}_{i}$, where $i=1, \ldots, m$. Let $K$ be the simplicial complex associated to $\Sigma$. Let $\beta_{i}$ be the integral primitive generator of each ray $\rho_{i}$, define an integral $n \times m$ matrix $B:=\left[\mathrm{b}_{1} \beta_{1}, \ldots, \mathrm{b}_{m} \beta_{m}\right]$, and let $\mathrm{G}$ be the kernel of the induced map of tori $B: \mathrm{T} \rightarrow \mathrm{R}$. The toric Deligne-Mumford (DM) stack associated to a labeled fan $(\Sigma, b)$ is defined as the quotient stack $\mathcal{X}_{\Sigma, b}:=\left[X_{\Sigma_{K}} / G_{\mathbb{C}}\right]$, where $\mathrm{G}_{\mathbb{C}}$ is the complexification of $\mathrm{G}$.

A toric DM stack $\mathcal{X}_{\Sigma, \mathrm{b}}$ (or its labeled fan $(\Sigma, \mathrm{b})$ ) is complete if the fan is complete ie the union of cones is $\mathbb{R}^{n}$. A toric DM stack $\mathcal{X}_{\Sigma, \mathrm{b}}$ is a nonsingular toric variety if and only if the labels $b_{i}=1$ and the fan $\Sigma$ is nonsingular, ie for each maximal cone, the primitive generators of the rays in the cone, $\beta_{i_{1}}, \ldots, \beta_{i_{n}}$, form a $\mathbb{Z}$-basis. In this case, we call $(\Sigma, b)$ nonsingular. For a nonsingular and complete labeled fan, we have the following classical result.

Theorem 2.10 (Danilov [8], Jurkiewicz [19]) If $(\Sigma, b)$ is nonsingular and complete, then $H^{*}\left(\left[\mathcal{X}_{\Sigma_{K}} / G_{\mathbb{C}}\right] ; \mathbb{Z}\right) \cong \mathbb{Z}[K] /\left\langle u_{1}, \ldots, u_{n}\right\rangle$. Furthermore, it has no $\mathbb{Z}$ torison [8, Theorem 10.8].

In [3, Proposition 3.7], it is proved that the coarse moduli space (underlying algebraic variety) for $\left[X_{\Sigma_{K}} / G_{\mathbb{C}}\right]$ is exactly the toric variety $X_{\Sigma}$ given by the fan $\Sigma$; see also Cox [7]. ${ }^{1}$ Thus for more general cases, one still has an isomorphism with $\mathbb{Q}$-coefficients.

${ }^{1}$ Even if the toric orbifold is a nontrivial orbifold, its coarse moduli space could be a nonsingular toric variety. The simplest example of such a case may be the weighted projective space $\left[\mathbb{C} \mathbb{P}_{12}^{1}\right]=$ $\left[\mathbb{C}^{2} \backslash\{(0,0)\} / \mathbb{C}^{\times}\right]$where the action of $\mathbb{C}^{\times}$is weighted by $(1,2)$. Its coarse moduli space is simply $\mathbb{C P}^{1}$. 
Theorem 2.11 (Danilov [8]) If $(\Sigma, \mathrm{b})$ is complete, then

where $u_{j}=\sum_{i=1}^{m} B_{j i} x_{i}$.

$$
H^{*}\left(\left[X_{\Sigma_{K}} / G_{\mathbb{C}}\right] ; \mathbb{Q}\right) \cong \mathbb{Q}[K] /\left\langle u_{1}, \ldots, u_{n}\right\rangle,
$$

Proposition 2.9 and Remark 2.4 therefore imply the following corollary.

Corollary 2.12 If $K$ and $B$ are given by a complete labeled fan, then $H_{\mathrm{G}}^{*}\left(\mathcal{Z}_{K} ; \mathbb{Q}\right) \cong$ $\mathbb{Q}[K] /\left\langle u_{1}, \ldots, u_{n}\right\rangle$. If $K$ and $B$ are given by a complete and nonsingular labeled fan, then $H_{\mathrm{G}}^{*}\left(\mathcal{Z}_{K} ; \mathbb{Z}\right) \cong \mathbb{Z}[K] /\left\langle u_{1}, \ldots, u_{n}\right\rangle$.

Note that the underlying combinatorial structures for quasitoric orbifolds and toric DM stacks are both simplicial complexes. Any symplectic toric orbifold can be made into an algebraic one by taking the normal fans to the polytopes. However, not all quasitoric orbifolds can be made algebraic. A toric DM stack associated to a polytopal fan can be made into a symplectic toric orbifold but there is a toric DM stack associated to a nonpolytopal fan. Such a toric DM stack can not be realized even as a quasitoric orbifold.

In light of Theorem 3.3, and Corollaries 2.8 and 2.12, it is natural to ask the following question.

Question 2.13 When is $H_{\mathrm{G}}^{*}\left(\mathcal{Z}_{K} ; \mathbb{Z}\right)$ a quotient of the Stanley-Reisner ring for a general subgroup $\mathrm{G}$ ?

Our answer to this question is Theorem 5.1. Also we will see in Section 5 that when the $\mathrm{G}$-action is locally free and $H_{\mathrm{G}}^{*}\left(\mathcal{Z}_{K} ; \mathbb{Q}\right)$ is a quotient of Stanley-Reisner ring, then the dimension of $G$ must be maximal.

\section{The proof that $H_{\mathrm{G}}^{*}\left(\mathcal{Z}_{K} ; \mathbb{Z}\right) \cong \operatorname{Tor}_{\mathbb{Z}\left[\mathbb{R}^{*}\right]}^{*}(\mathbb{Z}[K], \mathbb{Z})$}

In this section, we prove that $H_{\mathrm{G}}^{*}\left(\mathcal{Z}_{K} ; \mathbb{Z}\right)$ is isomorphic to $\operatorname{Tor}_{\mathbb{Z}\left[\mathrm{R}^{*}\right]}^{*}(\mathbb{Z}[K], \mathbb{Z})$ as a graded module over $\mathbb{Z}\left[T^{*}\right]$. The idea of the proof, especially to use the homological machinery developed in [12], was communicated to us by Franz. Throughout, we will use terminology found in [12].

We will use the following notation consistently throughout this paper unless otherwise specified. 
Notation 3.1 Let $K$ be a simplicial complex on $[m]:=\{1, \ldots, m\}$ possibly with ghost vertices, ie there may be $i \in[m]$ such that $\{i\} \notin K$. Let $\mathcal{Z}_{K}$ be the associated moment angle complex with the standard torus $\mathrm{T}:=\mathrm{U}(1)^{m}$-action. Let $\mathfrak{t}:=\operatorname{Lie}(\mathrm{T})=\mathbb{R}^{m}$, and let $\mathrm{N}_{\mathrm{T}}=\mathbb{Z}^{m}$ be the kernel of the exponential map $\mathrm{t} \rightarrow \mathrm{T}$. Let $\mathrm{G} \subset \mathrm{T}$ be a (possibly disconnected) closed subgroup of dimension $m-n$ and let $\mathrm{R}:=\mathrm{T} / \mathrm{G}$ be the quotient torus. We identify $\mathrm{R} \cong \mathrm{U}(1)^{n}$ so that the quotient map $\mathrm{T} \rightarrow \mathrm{R}$ defines an integral $n \times m$ matrix $B$ which is viewed as the surjective linear map $t \rightarrow \mathfrak{r}:=$ Lie $R$.

Let $\mathbb{Z}\left[\mathrm{T}^{*}\right]:=H^{*}(B \mathrm{~T} ; \mathbb{Z})=\mathbb{Z}\left[x_{1}, \ldots, x_{m}\right]$ where $\left\{x_{j}\right\}$ is the standard basis of $\mathrm{N}_{\mathrm{T}}^{*}$ and let $\mathbb{Z}\left[\mathrm{R}^{*}\right]:=H^{*}(B \mathrm{R} ; \mathbb{Z})=\mathbb{Z}\left[u_{1}, \ldots, u_{n}\right]$ where $\left\{u_{i}\right\}$ is the standard basis of $\mathrm{N}_{\mathrm{R}}^{*}$. We regard $\mathbb{Z}\left[\mathrm{R}^{*}\right]$ as a subring of $\mathbb{Z}\left[\mathrm{T}^{*}\right]$ so that $u_{i}:=\sum_{j=1}^{m} B_{i j} x_{j}$. The Stanley-Reisner ring $\mathbb{Z}[K]$ is defined as the quotient of $\mathbb{Z}\left[\mathrm{T}^{*}\right]$ by the monomials corresponding to nonfaces of $K$.

Definition 3.2 $\mathrm{M}$ is an $H^{*}(B \mathrm{R} ; \mathbb{Z})$-module up to homotopy if it is a module over the reduced cobar construction of $H^{*}(\mathrm{R} ; \mathbb{Z})[13$, Section 4$]$. Note that $H^{*}(\mathrm{R} ; \mathbb{Z})$ is regarded as a coalgebra dual to the algebra $H_{*}(\mathrm{R} ; \mathbb{Z})$ which is canonically isomorphic to the exterior algebra of $\mathrm{N}_{\mathrm{R}}$.

Theorem 3.3 Under Notation 3.1, there is an isomorphism of graded $\mathbb{Z}$-modules

$$
\Theta_{\mathrm{R}}: H_{\mathrm{G}}^{*}\left(\mathcal{Z}_{K} ; \mathbb{Z}\right) \rightarrow \operatorname{Tor}_{\mathbb{Z}\left[\mathrm{R}^{*}\right]}^{*}(\mathbb{Z}[K], \mathbb{Z}),
$$

where the cohomological grading on the right hand side is given in Definition 2.2. Furthermore there is a commutative diagram of graded $\mathbb{Z}$-modules

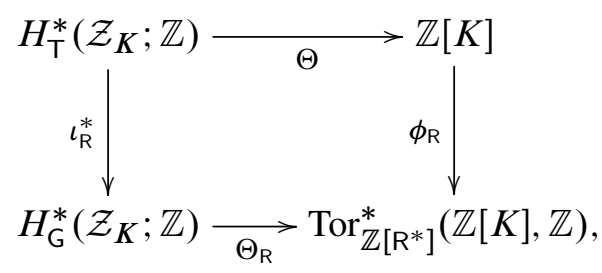

where $\phi_{\mathrm{R}}$ is the obvious map induced from the inclusion of Koszul complexes, $\iota_{\mathrm{R}}^{*}$ is the pullback of the quotient map $\iota_{\mathrm{R}}: E \mathrm{~T} \times_{\mathrm{G}} \mathcal{Z}_{K} \rightarrow E \mathrm{~T} \times_{\mathrm{T}} \mathcal{Z}_{K}$ and $\Theta$ is the isomorphism (1) of Section 2. In particular, $\Theta_{\mathrm{R}}$ is an isomorphism of graded rings if $\iota_{\mathrm{R}}^{*}$ is surjective.

Proof We suppress the coefficient ring $\mathbb{Z}$. Given a map $p: Y \rightarrow B \mathrm{R}$, the twisted tensor product $C^{*}(Y) \otimes^{\mathrm{R}} H^{*}(\mathrm{R})$ is defined by [12, (5.7)]. Franz [12, Proposition 5.2] states that there is a quasi-isomorphism of differential graded $(\mathrm{dg}) H_{*}(\mathrm{R})$-modules

$$
\Phi_{Y}^{*}: C^{*}(Y) \otimes^{\mathrm{R}} H^{*}(\mathrm{R}) \rightarrow C^{*}\left(Y \times{ }^{B \mathrm{R}} E \mathrm{R}\right),
$$


where $Y \times{ }^{B \mathrm{R}} E \mathrm{R}$ is a pullback of $E \mathrm{R} \rightarrow B \mathrm{R}$ along $p: Y \rightarrow B \mathrm{R}$.

Let $Y:=E \mathrm{R} \times{ }_{\mathrm{R}}\left(E \mathrm{~T} \times_{\mathrm{G}} \mathcal{Z}_{K}\right)$ and $p: Y \rightarrow B \mathrm{R}$ be defined as the composition of

$$
E \mathrm{R} \times \times_{\mathrm{R}}\left(E \mathrm{~T} \times \times_{\mathrm{G}} \mathcal{Z}_{K}\right) \underset{\mathrm{qR}_{\mathrm{R}}}{\longrightarrow} E \mathrm{~T} \times \times_{\mathrm{T}} \mathcal{Z}_{K} \longrightarrow B \mathrm{~T} \longrightarrow B \mathrm{R},
$$

where $\mathrm{q}_{\mathrm{R}}$ is the projection to the $2^{\text {nd }}$ and $3^{\text {rd }}$ components, the second map is the projection to the $1^{\text {st }}$ component and the last map is a classifying map for $B: \mathrm{T} \rightarrow \mathrm{R}$. We observe that $p$ is obtained by taking the quotient of

$$
E \mathrm{R} \times\left(E \mathrm{~T} \times{ }_{\mathrm{G}} \mathcal{Z}_{K}\right) \rightarrow E \mathrm{~T} \times{ }_{\mathrm{G}} \mathcal{Z}_{K} \rightarrow E \mathrm{~T} / \mathrm{G} \rightarrow E \mathrm{R}
$$

by the free actions of $\mathrm{R}$ on each space. This implies that $Y \times{ }^{B \mathrm{R}} E \mathrm{R}=E \mathrm{R} \times\left(E \mathrm{~T} \times{ }_{\mathrm{G}} \mathcal{Z}_{K}\right)$.

Now [12, Proposition 5.2] states that we have the quasi-isomorphism of $\operatorname{dg} H_{*}(\mathrm{R})-$ modules:

$$
\Phi_{Y}^{*}: C^{*}\left(E \mathrm{R} \times_{\mathrm{R}}\left(E \mathrm{~T} \times_{\mathrm{G}} \mathcal{Z}_{K}\right)\right) \otimes^{\mathrm{R}} H^{*}(\mathrm{R}) \rightarrow C^{*}\left(E \mathrm{R} \times\left(E \mathrm{~T} \times_{\mathrm{G}} \mathcal{Z}_{K}\right)\right)
$$

The homology of the right hand side is $H_{\mathrm{G}}^{*}\left(\mathcal{Z}_{K}\right)$. On the left hand side, since $\mathrm{R}$ acts on $E \mathrm{~T} \times{ }_{\mathrm{G}} \mathcal{Z}_{K}$ freely, the fibers of $\mathrm{q}_{\mathrm{R}}$ are $E \mathrm{R}$ and therefore it induces a quasi-isomorphism of $H^{*}(B \mathrm{~T})$-modules up to homotopy

$$
\mathrm{q}_{\mathrm{R}}^{*}: C^{*}\left(E \mathrm{~T} \times_{\mathrm{T}} \mathcal{Z}_{K}\right) \rightarrow C^{*}\left(E \mathrm{R} \times_{\mathrm{R}}\left(E \mathrm{~T} \times_{\mathrm{G}} \mathcal{Z}_{K}\right)\right),
$$

ie it is a homomorphism of $\mathrm{dg} C^{*}(B \mathrm{R})$-modules such that after taking homology, it becomes an isomorphism of $H^{*}(B \mathrm{R})$-modules. Franz [13, Theorem 1.1] implies that $C^{*}\left(E \mathrm{~T} \times \times_{\mathrm{T}} \mathcal{Z}_{K}\right)$ is formal as a $H^{*}(B \mathrm{~T})$-module up to homotopy, ie there is a sequence of quasi-isomorphisms connecting $C^{*}\left(E \mathrm{~T} \times_{\mathrm{T}} \mathcal{Z}_{K}\right)$ to $H^{*}\left(E \mathrm{~T} \times_{\mathrm{T}} \mathcal{Z}_{K}\right)$ as dg modules over the reduced cobar construction of $H^{*}(\mathrm{~T})$, and therefore as dg modules over the reduced cobar construction of $H^{*}(\mathrm{R})$. Since the operation to take the twisted tensor product $\otimes^{\mathrm{R}} H^{*}(B \mathrm{R})$ and the homology of it preserves quasi-isomorphisms of $H^{*}(B \mathrm{R})$-modules up to homotopy (cf McCleary [24, Theorem 8.20]), the map $\mathrm{q}_{\mathrm{R}}^{*}$ induces a quasi-isomorphism

$$
\widetilde{\mathrm{q}}_{\mathrm{R}}^{*}: C^{*}\left(E \mathrm{R} \times \times_{\mathrm{R}}\left(E \mathrm{~T} \times_{\mathrm{G}} \mathcal{Z}_{K}\right)\right) \otimes^{\mathrm{R}} H^{*}(\mathrm{R}) \rightarrow C^{*}\left(E \mathrm{~T} \times_{\mathrm{T}} \mathcal{Z}_{K}\right) \otimes^{\mathrm{R}} H^{*}(\mathrm{R}),
$$

and there is a sequence of quasi-isomorphisms connecting $C^{*}\left(E \mathrm{~T} \times \times_{\mathrm{T}} \mathcal{Z}_{K}\right) \otimes^{\mathrm{R}} H^{*}(\mathrm{R})$ and $H^{*}\left(E \mathrm{~T} \times \times_{\mathrm{T}} \mathcal{Z}_{K}\right) \otimes{ }^{\mathrm{R}} H^{*}(\mathrm{R})$. Since the homology of $H^{*}\left(E \mathrm{~T} \times{ }_{\mathrm{T}} \mathcal{Z}_{K}\right) \otimes{ }^{\mathrm{R}} H^{*}(\mathrm{R})$ is $\operatorname{Tor}_{\mathbb{Z}\left[\mathrm{R}^{*}\right]}^{*}(\mathbb{Z}[K], \mathbb{Z})$, we obtain the isomorphism $\Theta_{\mathrm{R}}$. We summarize all in the diagram: 


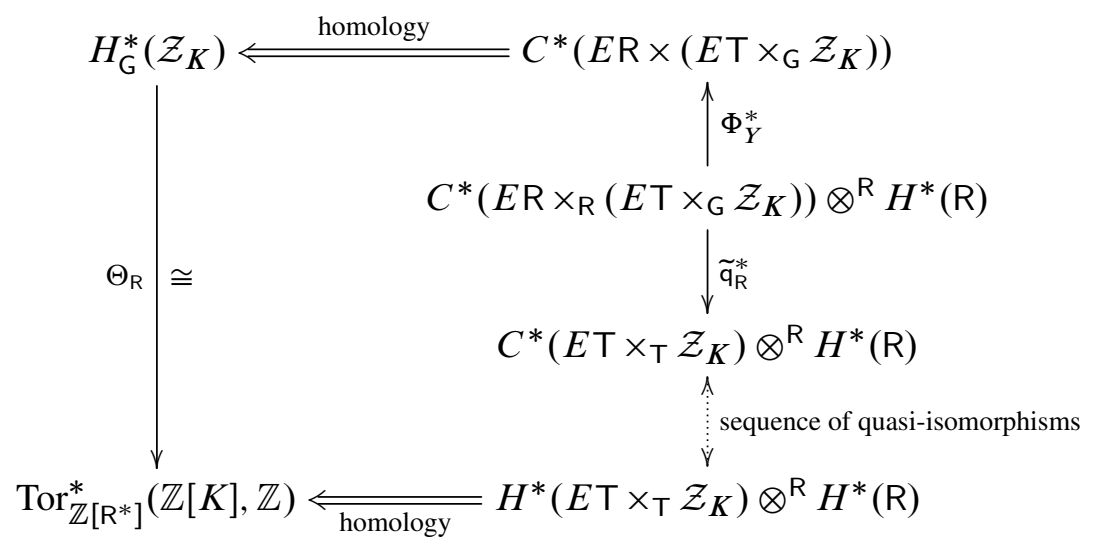

The right vertical maps gives a sequence of quasi-isomorphisms of $\mathrm{dg} H_{*}(\mathrm{R})$-modules and at the both ends, we have the desired graded $\mathbb{Z}$-modules after taking homology.

To prove the commutativity of diagram (3), first note that $\Theta$ coincides with $\Theta_{R}$ when $R=\mathbb{1}$, where $\mathbb{1}$ is the trivial group. Consider the map $\varphi: R \rightarrow \mathbb{1}$. Since it satisfies the condition in [12, Proposition 4.11], the naturality stated in [12, Proposition 5.2] implies that the following diagram commutes:

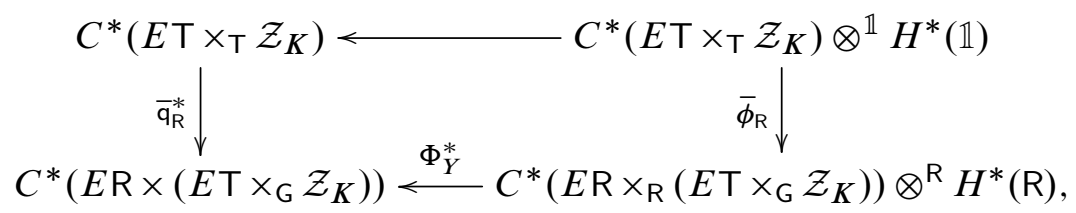

where $\overline{\mathrm{q}}_{\mathrm{R}}^{*}$ is the pullback of the projection $\overline{\mathrm{q}}_{\mathrm{R}}: E \mathrm{R} \times\left(E \mathrm{~T} \times_{\mathrm{G}} \mathcal{Z}_{K}\right) \rightarrow E \mathrm{~T} \times \times_{\mathrm{T}} \mathcal{Z}_{K}$ and $\bar{\phi}_{\mathrm{R}}$ is the map induced by $\mathrm{q}_{\mathrm{R}}$ and $\varphi: \mathrm{R} \rightarrow \mathbb{1}$. After taking the homology, $\overline{\mathrm{q}}_{\mathrm{R}}^{*}$ and $\bar{\phi}_{\mathrm{R}}$ naturally coincide with $\iota_{\mathrm{R}}^{*}$ and $\phi_{\mathrm{R}}$ respectively and the resulting diagram extends to the commutative diagram (3) since the rest of the right vertical maps in the diagram (4) are also natural with respect to $\varphi: R \rightarrow \mathbb{1}$.

Lastly, if $\iota_{\mathrm{R}}^{*}$ is surjective, the commutative diagram (3) makes $\Theta_{\mathrm{R}}$ an isomorphism of rings since the other three maps are ring homomorphism.

Remark 3.4 Let $X$ be any topological space with a T-action where $C^{*}\left(E \mathrm{~T} \times_{\mathrm{T}} X ; \mathbb{Z}\right)$ is formal as a $H^{*}(B \mathrm{~T} ; \mathbb{Z})$-module up to homotopy. Then Theorem 3.3 also holds for $X$. Namely, the above proof can be identically applied to this case and gives the isomorphism $\Theta_{\mathrm{R}}: H_{\mathrm{G}}^{*}(X ; \mathbb{Z}) \cong \operatorname{Tor}_{\mathbb{Z}\left[\mathrm{R}^{*}\right]}^{*}\left(H_{\mathrm{T}}^{*}(X ; \mathbb{Z}), \mathbb{Z}\right)$.

In terms of toric orbifolds discussed in Section 2, we have the following corollary. 
Corollary 3.5 Let $\mathcal{X}$ is a quasitoric orbifold or an effective toric Deligne-Mumford stack with associated simplicial complex $K$, and let $\mathrm{R}$ and $\mathrm{T}$ be the tori given in the notation of Section 2. Then there is an isomorphism of graded modules over $\mathbb{Z}\left[\mathrm{T}^{*}\right]$

$$
H^{*}(\mathcal{X} ; \mathbb{Z}) \cong \operatorname{Tor}_{\mathbb{Z}\left[\mathrm{R}^{*}\right]}^{*}(\mathbb{Z}[K], \mathbb{Z}) .
$$

Remark 3.6 Theorem 3.3 (or Corollary 3.5) generalizes several results that have been proved.

(i) In case $\mathrm{G}=\mathrm{T}$, Theorem 3.3 states that the T-equivariant cohomology of $\mathcal{Z}_{K}$ is the Stanley-Reisner ring of $K$, which is well-known; cf [5]. In case $G=1$, then one recovers that the ordinary cohomology of $\mathcal{Z}_{K}$ is the Tor-algebra of $\mathbb{Z}[K]$ over $\mathbb{Z}\left[T^{*}\right][5$, Theorem 7.6]. One may therefore view this result as interpolating between these extreme cases.

(ii) If $\mathcal{Z}_{K} / G$ is a quasitoric manifold, then one recovers [5, Theorem 7.37].

(iii) When $X_{\Sigma}:=X_{\Sigma_{K}} / \mathrm{G}_{\mathbb{C}}$ is the coarse moduli for a toric orbifold, one recovers [13, Theorem 1.2]:

$$
H^{*}\left(X_{\Sigma} ; \mathbb{Q}\right) \cong H_{\mathrm{G}_{\mathbb{C}}}^{*}\left(X_{\Sigma_{K}} ; \mathbb{Q}\right)=H_{\mathrm{G}}^{*}\left(\mathcal{Z}_{K} ; \mathbb{Q}\right) \cong \operatorname{Tor}_{\mathbb{Z}\left[\mathrm{R}^{*}\right]}^{*}(\mathbb{Z}[K], \mathbb{Z}) \otimes \mathbb{Q}
$$

\section{Basics from commutative algebra}

In this section, we collect some definitions and basic properties of graded modules over a polynomial ring and discuss the relations among them. Throughout this section, $R$ will be a polynomial ring in variables $u_{1}, \ldots, u_{n}$ (generated in degree 2) over $k=\mathbb{Z}$ or $\mathbb{Q}$, and $M$ will be a graded $R$-module (though not necessarily finitely generated). We will denote the ideal of $R$ generated by polynomials of positive degree by $R_{+}$.

Below we give brief names to several properties of $R$-modules so that we can refer to them later.

Definition 4.1 For $M$ and $R$ as above, one says that

(k1) $\quad M$ is free over $R$ if $M \cong \bigoplus_{e \in E} R \cdot e$ and $R \cdot e \cong R$ as graded $R$-modules where $E$ is a subset of $M$,

(k2) $\quad M$ is flat over $R$ if $\operatorname{Tor}_{>0}^{R}(M, N)=0$ for any (finitely generated) module $N$,

(k3) $M$ is torsion-free over $R$ if there is no torsion over $R$ ( $x \in M$ is a torsion element over $R$ if $x$ is in the kernel of the multiplication map $\cdot r: M \rightarrow M$ for some nonzero $r \in R$ ),

(k4) $\quad M$ is a big Cohen-Macaulay $R$-module if $\operatorname{Tor}_{1}^{R}(M, k)=0$.

In general, (k1) implies (k2), and (k2) implies both (k3) and (k4). 
We will see that in the case of interest to us, $(\mathrm{k} 4)$ has the usual meaning in terms of regular sequences; see Proposition 4.6.

Definition 4.2 A nonzero element $r \in R$ is $M$-regular if $0 \longrightarrow M \stackrel{r}{\longrightarrow} M$ is exact. A sequence of elements $f_{1}, \ldots, f_{c} \subset R$ is an $M$-regular sequence if, for each $i \leq c, f_{i}$ is $\left(M /\left(f_{1}, \ldots, f_{i-1}\right) M\right)$-regular.

Remark 4.3 We call the condition (k4) in Definition 4.1 big Cohen-Macaulay because $\operatorname{Tor}_{1}^{R}(M, k)=0$ is the same as saying that there exists a system of parameters of $R_{+}$ (namely, the variables of $R$; see Corollary 4.12) that is an $M$-regular sequence. The 'big' terminology is a reference to the fact that $M$ need not be finitely generated; see Bruns and Herzog [4, Chapter 8]. ${ }^{2}$

Definition 4.4 Let $I$ be an ideal of $R$ such that $M \neq I M$. One defines the grade of $I$ on $M$ by

$$
\operatorname{grade}(I, M):=\min \left\{i \mid \operatorname{Ext}_{R}^{i}(R / I, M) \neq 0\right\} .
$$

If $M=I M$, one sets $\operatorname{grade}(I, M)=\infty$. When $M$ is finitely generated over $R$, this definition is the usual definition of the grade of $I$ on $M$ and it is the length of maximal $M$-regular sequence in $I$ [4].

If $I=R_{+}$is the ideal generated by the positive degree elements of $R$, the grade of $I$ on $M$ is called the depth of $M$ over $R$, and is denoted $\operatorname{depth}_{R} M:=\operatorname{grade}(I, M)$.

Our goal for this section is to show that $\operatorname{depth}_{R}(M)$ is the length of the longest $M-$ regular sequence in $R_{+}$when $M$ is only assumed to be finitely generated over $S$, where $f: R \rightarrow S$ is a ring homomorphism and the $R$-action on $M$ is induced by $f$. This is recorded in the following proposition.

Proposition 4.5 Let $S=k\left[x_{1}, \ldots, x_{m}\right], M$ be a finitely generated graded $S$-module, and $\varphi: R \rightarrow S$ be a graded ring homomorphism (so that $M$ is hence a graded $R-$ module via $\varphi$ ). Then all maximal $M$-regular sequences in $R_{+}$have the same length $\operatorname{depth}_{R}(M)$.

This proposition is a special case of the following proposition, whose proof will come after some lemmas.

\footnotetext{
${ }^{2}$ In the reference [4], it is mentioned that the existence of a big Cohen-Macaulay module over a local ring is an open problem. This question is not interesting for $R$, since it is a (nonlocal) CohenMacaulay ring.
} 
Proposition 4.6 Let $\varphi: R \rightarrow S$ be a homomorphism of Noetherian rings, $M$ a finitely generated $S$-module and $I$ an ideal of $R$ with $I M \neq M$. Then grade $(I, M)$ agrees with the length of all maximal $M$-regular sequences in $I$.

The fact that allows one to extend the usual finitely generated setup to the generality above is the following lemma.

Lemma 4.7 Let $\varphi: R \rightarrow S$ be a homomorphism of graded Noetherian rings, $M$ a finitely generated $S$-module and $N$ a finitely generated $R$-module. Then we have $\operatorname{Hom}_{R}(N, M)=0$ if and only if $\operatorname{Ann}_{R}(N)$ contains an $M$-regular element.

Remark 4.8 Before starting on the proof, let us remark that in the setup of the lemma the set of associated primes of $M$ over $R$ is finite, even though $M$ may not be finitely generated over $R$; see Matsumura [23, Exercise 6.7]. Indeed, one sees this by taking a primary decomposition of $M$ over $S$, which is also a primary decomposition over $R$, since the $R$-annihilator of an $S$-module is the preimage of the annihilator in $S$ (and the inverse image of a primary ideal is primary).

Proof of Lemma 4.7 Suppose that $x \in \operatorname{Ann}_{R}(N)$ is $M$-regular. Then for any $\psi \in \operatorname{Hom}_{R}(N, M)$, we have

$$
x \psi(n)=\psi(x n)=\psi(0)=0 \text { for all } n \in N .
$$

Since $x$ is $M$-regular, we have $\psi(n)=0$.

Now assume that $\operatorname{Ann}_{R}(N)$ does not contain an $M$-regular element. That is, all elements of $\operatorname{Ann}_{R}(N)$ are zero divisors on $M$. As mentioned in Remark 4.8, the set of associated primes of $M$ over $R$ is finite. Since $\operatorname{Ann}_{R}(N)$ consists of zero divisors, it is contained in the (finite) union of all associated primes of $M$. Therefore, we can apply the Prime Avoidance Lemma to get $\operatorname{Ann}_{R}(N) \subset \mathfrak{p}$ for some associated prime $\mathfrak{p}$ of $M$ over $R$. We then have the nontrivial map

$$
N_{\mathfrak{p}} \rightarrow N_{\mathfrak{p}} / \mathfrak{p} N_{\mathfrak{p}} \rightarrow k(\mathfrak{p}) \hookrightarrow M_{\mathfrak{p}},
$$

where $k(\mathfrak{p})$ denotes the residue field $R_{\mathfrak{p}} / \mathfrak{p} R_{\mathfrak{p}}$. Note that the first map is the canonical projection, the second is projection onto a one-dimensional subspace of $N_{\mathfrak{p}} / \mathfrak{p} N_{\mathfrak{p}}$, and the inclusion comes from localizing the inclusion $R / \mathfrak{p} \hookrightarrow M$ (which exists because $\mathfrak{p}$ is an associated prime of $M)$ at $\mathfrak{p}$. Thus, since $N$ is finitely generated over $R$, $\operatorname{Hom}_{R}(N, M)_{\mathfrak{p}}=\operatorname{Hom}_{R_{\mathfrak{p}}}\left(N_{\mathfrak{p}}, M_{\mathfrak{p}}\right) \neq 0$, which gives $\operatorname{Hom}_{R}(N, M) \neq 0$.

We also record without proof a basic fact from the homological algebra of commutative rings. 
Lemma 4.9 [4, Lemma 1.2.4] Let $\varphi: R \rightarrow S$ be a homomorphism of Noetherian rings, $M$ a finitely generated $S$-module and $N$ a finitely generated $R$-module. If $\left(x_{1}, \ldots, x_{r}\right)$ is a regular sequence in $\operatorname{Ann}_{R}(N)$ for $M$, then

$$
\operatorname{Hom}_{R}\left(N, M /\left(x_{1}, \ldots, x_{r}\right) M\right)=\operatorname{Ext}_{R}^{r}(N, M) .
$$

Proof of Proposition 4.6 Let $\left(x_{1}, \ldots, x_{r}\right)$ be a maximal $M$-regular sequence in $I$. By Lemma 4.9,

$$
\operatorname{Ext}_{R}^{i}(R / I, M) \cong \operatorname{Hom}_{R}\left(R / I, M /\left(x_{1}, \ldots, x_{i}\right) M\right) .
$$

If $i<r$, then $x_{i+1}$ is $M /\left(x_{1}, \ldots, x_{i}\right) M$-regular. Therefore $\operatorname{Ext}_{R}^{i}(R / I, M)=0$ by Lemma 4.7. Since $\left(x_{1}, \ldots, x_{r}\right)$ is maximal, $I$ doesn't contain any $M /\left(x_{1}, \ldots, x_{r}\right) M-$ regular elements. Thus $\operatorname{Ext}_{R}^{r}(R / I, M) \neq 0$. This proves the claim.

One has the following well known characterization of (k4) in terms of $M$-regular sequences due to Serre [27, Chapter IV.A].

Proposition 4.10 Let $u_{1}, \ldots, u_{n}$ be a homogeneous minimal generating set of $R_{+}$. Suppose that $R$ and $M$ satisfy the hypotheses of Proposition 4.5. Then the following properties are equivalent:

(a) $H_{p}(\boldsymbol{u}, M)=0$ for $p \geq 1$.

(b) $H_{1}(\boldsymbol{u}, M)=0$.

(c) The sequence $u_{1}, \ldots, u_{n}$ is $M$-regular.

Here, $H_{p}(\boldsymbol{u}, M)$ denotes the $p^{\text {th }}$ Koszul homology of the sequence $u_{1}, \ldots, u_{n}$ with coefficients in $M$.

Proof The proof that appears in Serre [27, Chapter IV.A] uses standard techniques of the Koszul complex which hold even for modules which are not finitely generated over $R$, together with Nakayama's lemma for finitely generated modules over a Noetherian local ring. Since a version of Nakayama's lemma holds for graded modules that satisfy our hypothesis, Serre's original argument remains valid.

Corollary 4.11 Let $u_{1}, \ldots, u_{n}$ be a homogeneous minimal generating set of $R_{+}$, and suppose that $R$ and $M$ satisfy the hypothesis of Proposition 4.6. Then $\operatorname{Tor}_{1}^{R}(M, k)=0$ if and only if $\left(u_{1}, \ldots, u_{n}\right)$ is a regular sequence for $M$.

Proof The Koszul complex on $u_{1}, \ldots, u_{n}$ resolves $k$ over $R$, and hence one can use its homology to compute the Tor modules. Now appeal to the previous proposition. 
Propositions 4.11 and 4.5 show that in the setup of Proposition 4.5, big CohenMacaulayness of $M$ has the usual meaning in terms of the maximal length of an $M$-regular sequence.

Corollary 4.12 In the setup of Proposition 4.5, one has that $\operatorname{Tor}_{1}^{R}(M, k)=0$ if and only if $\operatorname{depth}_{R}(M)=n$.

\section{Properties of $\mathbb{Z}[K]$ as an algebra over $\mathbb{Z}\left[R^{*}\right]$}

In this section, we start with the characterization of big Cohen-Macaulayness (k4), and then discuss freeness $(\mathrm{k} 1)$ and torsion-freeness $(\mathrm{k} 3)$, of $\mathbb{Z}[K]$ as a ring over $\mathbb{Z}\left[\mathrm{R}^{*}\right]$. In the rest of the paper, we use Notation 3.1 unless otherwise specified.

\subsection{Big Cohen-Macaulayness}

The following theorem is a variant of Franz and Puppe [15, Theorem 1.1] and Franz [14, Lemma 5.1]. The differences are that the T-CW complex $E \mathrm{G} \times_{\mathrm{G}} \mathcal{Z}_{K}$ is not finite and that we consider the cohomology of the quotient stack $\left[\mathcal{Z}_{K} / \mathrm{G}\right]$ instead of the one of the underlying topological space $\mathcal{Z}_{K} / \mathrm{G}$.

Theorem 5.1 Let $\iota_{\mathrm{R}}: E \mathrm{~T} \times_{\mathrm{G}} \mathcal{Z}_{K} \rightarrow E \mathrm{~T} \times_{\mathrm{T}} \mathcal{Z}_{K}$ be the quotient map by the R-action. The following are equivalent:

(i) $\mathbb{Z}[K]$ is big Cohen-Macaulay over $\mathbb{Z}\left[\mathrm{R}^{*}\right]$, ie $\operatorname{Tor}_{1}^{\mathbb{Z}\left[\mathrm{R}^{*}\right]}(\mathbb{Z}[K], \mathbb{Z})=0$

(ii) $\iota_{\mathrm{R}}^{*}: H_{\mathrm{T}}^{*}\left(\mathcal{Z}_{K} ; \mathbb{Z}\right) \rightarrow H_{\mathrm{G}}^{*}\left(\mathcal{Z}_{K} ; \mathbb{Z}\right)$ is surjective

(iii) $H_{\mathrm{G}}^{\text {odd }}\left(\mathcal{Z}_{K} ; \mathbb{Z}\right)=0$

Proof Since $\operatorname{Tor}_{0}^{\mathbb{Z}\left[\mathrm{R}^{*}\right]}(\mathbb{Z}[K], \mathbb{Z})=\mathbb{Z}[K] /\left\langle u_{1}, \ldots, u_{n}\right\rangle$, the commutative diagram (3) in Theorem 3.3 implies that (ii) is equivalent to the vanishing of $\operatorname{Tor}_{i>0}^{\mathbb{Z}\left[\mathrm{R}^{*}\right]}(\mathbb{Z}[K], \mathbb{Z})$, which is actually equivalent to (i) by [15, Proposition 2.3]. (ii) implies (iii) because $H_{\top}^{*}\left(\mathcal{Z}_{K} ; \mathbb{Z}\right)$ has only even degree classes. Now (iii) implies that the Serre spectral sequence for the Borel construction for the residual R-action for $E \mathrm{~T} \times_{\mathrm{G}} \mathcal{Z}_{K}$ degenerates at $E_{2}$ level and hence the pullback of the fiber inclusion $E \mathrm{~T} \times_{\mathrm{G}} \mathcal{Z}_{K} \hookrightarrow$ $E \mathrm{R} \times{ }_{\mathrm{R}}\left(E \mathrm{~T} \times{ }_{\mathrm{G}} \mathcal{Z}_{K}\right)$ is surjective. This pullback can be identified as $\iota_{\mathrm{R}}^{*}$ and thus (iii) implies (ii); see also [14, Lemma 5.1] and its proof.

Again it is worth noting that Theorem 5.1 holds for any T-space $X$ that satisfies the formality hypothesis in Remark 3.4, as well as $H_{\top}^{\text {odd }}(X ; \mathbb{Z})$. 
Remark 5.2 By Theorem 3.3, $\iota_{\mathrm{R}}^{*}$ is surjective if and only if

$$
\Theta: H_{\mathrm{G}}^{*}\left(\mathcal{Z}_{K}, \mathbb{Z}\right) \cong \mathbb{Z}[K] /\left\langle u_{1}, \ldots, u_{n}\right\rangle .
$$

Remark 5.3 Theorem 5.1 holds after replacing $\mathbb{Z}$ by $\mathbb{Q}$.

Corollary 5.4 If $\mathcal{X}=\left[\mathcal{Z}_{K} / \mathrm{G}\right]$ is a quasitoric orbifold or effective toric DeligneMumford stack, then the following are equivalent:

(i) $\operatorname{Tor}_{1}^{\mathbb{Z}\left[\mathrm{R}^{*}\right]}(\mathbb{Z}[K], \mathbb{Z})=0$

(ii) $H^{*}(\mathcal{X} ; \mathbb{Z})$ is the quotient of $\mathbb{Z}[K]$ by $u_{1}, \ldots, u_{n}$

(iii) $H^{\text {odd }}(\mathcal{X} ; \mathbb{Z})=0$

\subsection{Freeness}

The following theorem is analogous to [14, Lemma 6.1].

Proposition 5.5 $\mathbb{Z}[K]$ is free over $\mathbb{Z}\left[\mathrm{R}^{*}\right]$ if and only if $H_{\mathrm{G}}^{*}\left(\mathcal{Z}_{K} ; \mathbb{Z}\right)$ has no $\mathbb{Z}$-torison and is concentrated in even degree.

Proof If $H_{\mathrm{G}}^{*}\left(\mathcal{Z}_{K} ; \mathbb{Z}\right)$ is concentrated in even degree, then by Theorem 5.1 we have that $\iota_{\mathrm{R}}^{*}: H_{\mathrm{T}}^{*}\left(\mathcal{Z}_{K} ; \mathbb{Z}\right) \rightarrow H_{\mathrm{G}}^{*}\left(\mathcal{Z}_{K} ; \mathbb{Z}\right)$ is surjective. The surjectivity implies that $H_{\mathrm{G}}^{r}\left(\mathcal{Z}_{K} ; \mathbb{Z}\right)$ has finite rank for each $r$ and is a finitely generated free $\mathbb{Z}$-module if it has no $\mathbb{Z}$-torison. The Leray-Hirsch theorem (cf Hatcher [16, Theorem 4D.1]) can be applied to the fiber bundle $E \mathrm{R} \times \times_{\mathrm{R}}\left(E \mathrm{~T} \times_{\mathrm{G}} \mathcal{Z}_{K}\right) \rightarrow B \mathrm{R}$ where the pullback along the fiber $E \mathrm{~T} \times_{\mathrm{G}} \mathcal{Z}_{K}$ can be identified with $\iota_{\mathrm{R}}^{*}$ and therefore we have an isomorphism $\mathbb{Z}\left[\mathrm{R}^{*}\right] \otimes_{\mathbb{Z}} H_{\mathrm{G}}^{*}\left(\mathcal{Z}_{K} ; \mathbb{Z}\right) \cong \mathbb{Z}[K]$ of $\mathbb{Z}\left[\mathrm{R}^{*}\right]$-modules. Since $H_{\mathrm{G}}^{*}\left(\mathcal{Z}_{K} ; \mathbb{Z}\right)$ is a free $\mathbb{Z}$-module, $\mathbb{Z}[K]$ is a free $\mathbb{Z}\left[\mathrm{R}^{*}\right]$ module.

On the other hand, freeness of $\mathbb{Z}[K]$ over $\mathbb{Z}\left[\mathrm{R}^{*}\right]$ implies $\operatorname{Tor}_{1}^{\mathbb{Z}\left[\mathrm{R}^{*}\right]}(\mathbb{Z}[K], \mathbb{Z})=0$ and so $\iota_{\mathrm{R}}^{*}$ is surjective by Theorem 5.1. Thus $H_{\mathrm{G}}^{*}\left(\mathcal{Z}_{K}, \mathbb{Z}\right) \cong H_{\mathrm{T}}^{*}\left(\mathcal{Z}_{K}, \mathbb{Z}\right) \otimes_{\mathbb{Z}\left[\mathrm{R}^{*}\right]} \mathbb{Z}$. By freeness, we can write $H_{\mathrm{T}}^{*}\left(\mathcal{Z}_{K} ; \mathbb{Z}\right) \cong \bigoplus_{e \in E} \mathbb{Z}\left[\mathrm{R}^{*}\right] e$ as a free $\mathbb{Z}\left[\mathrm{R}^{*}\right]$-module, where the $e$ are even degree classes. Then $H_{\mathrm{T}}^{*}\left(\mathcal{Z}_{K} ; \mathbb{Z}\right) \otimes_{\mathbb{Z}\left[\mathrm{R}^{*}\right]} \mathbb{Z} \cong \bigoplus_{e \in E} \mathbb{Z} e$. Thus there is no $\mathbb{Z}$-torison and no odd degree classes.

The same proof as above proves the following theorem; see also Remark 5.3.

Proposition 5.6 $\mathbb{Q}[K]$ is free over $\mathbb{Q}\left[\mathrm{R}^{*}\right]$ if and only if $H_{\mathrm{G}}^{*}\left(\mathcal{Z}_{K} ; \mathbb{Q}\right)$ has no odd degree classes. 
With this proposition, together with the local freeness of the $\mathrm{G}$-action, we can also prove the following lemma.

Lemma 5.7 Suppose that the $\mathrm{G}$-action on $\mathcal{Z}_{K}$ is locally free. If $H_{\mathrm{G}}^{*}\left(\mathcal{Z}_{K} ; \mathbb{Q}\right)$ is concentrated in even degree, then $\mathbb{Q}[K]$ is finitely generated over $\mathbb{Q}\left[\mathrm{R}^{*}\right]$.

Proof Since the $\mathrm{G}$-action on the smooth variety $X_{\Sigma_{K}}$ defined in Section 2.5 is locally free, we have the differentiable orbifold $\left[X_{\Sigma_{K}} / \mathrm{G}\right]$. By the construction of de Rham cohomology for differentiable orbifolds, cf Adem, Leida and Ruan [1, Section 2.1], $H^{*}\left(\left[X_{\Sigma_{K}} / \mathrm{G}\right] ; \mathbb{R}\right)$ is finite-dimensional. Since $\mathcal{Z}_{K} \hookrightarrow X_{\Sigma_{K}}$ is a T-equivariant deformation retract, $H_{\mathrm{G}}^{*}\left(\mathcal{Z}_{K} ; \mathbb{Q}\right)$ is also finite-dimensional. On the other hand, by Proposition 5.6, if $H_{\mathrm{G}}^{*}\left(\mathcal{Z}_{K} ; \mathbb{Q}\right)$ has no odd degree, then $\mathbb{Q}[K]$ is free over $\mathbb{Q}\left[\mathrm{R}^{*}\right]$. Since $H_{\mathrm{G}}^{*}\left(\mathcal{Z}_{K} ; \mathbb{Q}\right) \cong H_{\mathrm{T}}^{*}\left(\mathcal{Z}_{K} ; \mathbb{Q}\right) \otimes_{\mathbb{Q}\left[\mathrm{R}^{*}\right]} \mathbb{Q}$, the finiteness of $H_{\mathrm{G}}^{*}\left(\mathcal{Z}_{K} ; \mathbb{Q}\right)$ implies that $H_{\mathrm{T}}^{*}\left(\mathcal{Z}_{K} ; \mathbb{Q}\right)$ is finitely generated over $\mathbb{Q}\left[R^{*}\right]$.

\subsection{Torsion-freeness}

First we observe the following equivalence.

Lemma 5.8 $\mathbb{Z}[K]$ is torsion-free over $\mathbb{Z}\left[\mathrm{R}^{*}\right]$ if and only if $\mathbb{Z}[K] \otimes \mathbb{Q}$ is torsion-free over $\mathbb{Q}\left[R^{*}\right]$.

Proof Suppose that $f \neq 0$ is a torsion element in $\mathbb{Z}[K]$ over $\mathbb{Z}\left[\mathrm{R}^{*}\right]$, ie there is a nonzero element $g \in \mathbb{Z}\left[\mathrm{R}^{*}\right]$ such that $f g=0$ in $\mathbb{Z}[K]$. Since $\mathbb{Z}[K]$ is free over $\mathbb{Z}$, $f \neq 0$ in $\mathbb{Q}[K]$. Therefore $f$ is also a torsion in $\mathbb{Q}[K]$ over $\mathbb{Q}\left[\mathrm{R}^{*}\right]$. On the other hand, suppose that $f \neq 0$ is a torsion element of $\mathbb{Q}[K]$ over $\mathbb{Q}\left[\mathrm{R}^{*}\right]$. Let $g \in \mathbb{Q}\left[\mathrm{R}^{*}\right]$ be a nonzero element such that $f g=0$ in $\mathbb{Q}[K]$. Let $a$ be the product of denominators of the coefficients of $f$ and $b$ be the product of denominator of coefficients of $g$. Then the pair of $a f \in \mathbb{Z}[K]$ and $b g \in \mathbb{Z}\left[\mathrm{R}^{*}\right]$ gives a torsion element of $\mathbb{Z}[K]$ over $\mathbb{Z}\left[\mathrm{R}^{*}\right]$.

Theorem 5.9 If $H_{\mathrm{G}}^{*}\left(\mathcal{Z}_{K} ; \mathbb{Q}\right)$ is concentrated in even degree, then $\mathbb{Z}[K]$ is torsion-free over $\mathbb{Z}\left[\mathrm{R}^{*}\right]$.

Proof By Proposition 5.6, $\mathbb{Q}[K]$ is free over $\mathbb{Q}\left[\mathrm{R}^{*}\right]$, therefore it is torsion-free over $\mathbb{Q}\left[\mathrm{R}^{*}\right]$. We conclude that $\mathbb{Z}[K]$ is torsion-free over $\mathbb{Z}\left[\mathrm{R}^{*}\right]$ by Lemma 5.8.

If $\left[\mathcal{Z}_{K} / \mathrm{G}\right]$ is a quasitoric orbifold or a complete toric DM stack (Section 2$), H_{\mathrm{G}}^{*}\left(\mathcal{Z}_{K} ; \mathbb{Q}\right)$ is concentrated in even degree by Theorems 2.6 and 2.11. Thus we have the following:

Corollary 5.10 If $\left[\mathcal{Z}_{K} / \mathrm{G}\right]$ is a quasitoric orbifold or a complete toric DM stack, then $\mathbb{Z}[K]$ is torsion-free over $\mathbb{Z}\left[\mathrm{R}^{*}\right]$. 
Remark 5.11 The converse of Theorem 5.9 is not true. The direct product of weighted projective spaces is a complete toric DM stack and its cohomology has odd degree classes. See Example 6.2.

The following proposition shows that the vanishing of odd classes in $H_{\mathrm{G}}^{*}\left(\mathcal{Z}_{K} ; \mathbb{Q}\right)$ implies that the size of $\mathrm{G}$ is maximal and it is analogous to [14, Proposition 5.2].

Proposition 5.12 Let $n^{\prime}$ be the largest cardinality of a face of $K$. Suppose that $\mathrm{G}$ acts on $\mathcal{Z}_{K}$ locally freely. If $H_{\mathrm{G}}^{*}\left(\mathcal{Z}_{K} ; \mathbb{Q}\right)$ is concentrated in even degree, then $\operatorname{dim} \mathrm{R}=n^{\prime}$. Furthermore for a closed subgroup $\mathrm{G}_{1} \subset \mathrm{G}$ such that $\operatorname{dim} \mathrm{G}_{1}<\operatorname{dim} \mathrm{G}=m-n, \mathbb{Q}[K]$ has a torsion over $\mathbb{Q}\left[R_{1}^{*}\right]$, where $R_{1}:=T / G_{1}$.

Proof By Lemma 5.7, $\mathbb{Q}[K]$ is finitely generated over $\mathbb{Q}\left[\mathrm{R}^{*}\right]$ and hence over $\mathbb{Q}\left[\mathrm{R}_{1}^{*}\right]$. Moreover $\mathbb{Q}[K]$ is free over $\mathbb{Q}\left[\mathrm{R}^{*}\right]$ by Proposition 5.6 and so $A \mathrm{An}_{\mathbb{Q}\left[\mathrm{R}^{*}\right]} \mathbb{Q}[K]=0$. Thus we have

$$
n^{\prime}=\operatorname{dim} \mathbb{Q}[K]=\operatorname{dim} \mathbb{Q}\left[\mathrm{R}^{*}\right]=\operatorname{dim} \frac{\mathbb{Q}\left[\mathrm{R}_{1}^{*}\right]}{\operatorname{Ann}_{\mathbb{Q}\left[\mathrm{R}_{1}^{*}\right]} \mathbb{Q}[K]} .
$$

Thus $\operatorname{dim} \mathrm{R}=\operatorname{dim} \mathbb{Q}\left[\mathrm{R}^{*}\right]=n^{\prime}$ and $\operatorname{dim} \mathbb{Q}\left[\mathrm{R}_{1}^{*}\right]=\operatorname{dim} \mathrm{R}_{1}>\operatorname{dim} \mathrm{R}$, which implies $\operatorname{Ann}_{\mathbb{Q}\left[R_{1}^{*}\right]} \mathbb{Q}[K] \neq 0$.

Example 5.13 Let $\Delta$ be a $n$-dimensional rational bounded simple polytope in $\mathbb{R}^{n}=\mathfrak{r}^{*}$, where $\mathfrak{r}$ is the Lie algebra of $\mathrm{R}$ and $K_{\Delta}$ the corresponding simplicial complex. Let $\rho_{1}, \ldots, \rho_{m} \in \mathfrak{r}$ be the inward primitive normal vectors of the facets $H_{1}, \ldots, H_{m}$ of $\Delta$ and let $B=\left[\rho_{1} \cdots \rho_{m}\right]$ be an integral $n \times m$ matrix. Adopt the notation from Notation 3.1. The GKM description of the rational equivariant cohomology of the corresponding toric orbifold is essentially the description of the piecewise polynomial functions on the corresponding normal fan: Let $v_{1}, \ldots, v_{r}$ be the vertices of $\Delta$ and denote the edge between $v_{i}$ and $v_{j}$ by $(i, j)$. Then there is an isomorphism of $\mathbb{Q}\left[u_{1}, \ldots, u_{n}\right]$-modules

$\Phi: \mathbb{Q}\left[K_{\Delta}\right] \cong\left\{\left(f_{1}, \ldots, f_{r}\right) \mid \lambda_{i j}\right.$ divides $f_{i}-f_{j}$ for all edges $(i, j)$ of $\left.\Delta\right\} \subset \bigoplus_{i=1}^{r} \mathbb{Q}[\boldsymbol{u}]$, where $\mathbb{Q}[\boldsymbol{u}]:=\mathbb{Q}\left[u_{1}, \ldots, u_{n}\right]$ and $\lambda_{i j} \in \mathrm{r}^{*}$ is a vector generating the edge $(i, j)$ of $\Delta$. It is well-known that $\Phi\left(x_{i}\right)_{k}=0$ unless $v_{k}$ lies on $H_{i}$. Therefore, for each vertex $v_{k}=H_{i_{1}} \cap \cdots \cap H_{i_{n}}, \Phi\left(x_{i_{1}} \cdots x_{i_{n}}\right)_{l}=0$ unless $k=l$. Now suppose that we take a subgroup $\mathrm{G}_{1} \subset \mathrm{G}$ with $\operatorname{dim} \mathrm{G}_{1}<m-n$ and let $\mathrm{R}_{1}:=\mathrm{T} / \mathrm{G}_{1}$. Let $\mathfrak{r}_{1}$ be the Lie algebra of $\mathrm{R}_{1}$ and $u \in \mathfrak{r}_{1}^{*}$ a rational element such that $u \notin \mathfrak{r}^{*}$. Let $\Phi(u)_{k}=\sum_{i=1}^{n} a_{i} u_{i}$ so that $\Phi\left(u-\sum_{i=1}^{n} a_{i} u_{i}\right)_{k}=0$ and $u-\sum_{i=1}^{n} a_{i} u_{i} \neq 0$ since $u \notin \mathfrak{r}^{*}$. Therefore $\left(u-\sum_{i=1}^{n} a_{i} u_{i}\right) \cdot x_{i_{1}} \cdots x_{i_{n}}=0$, ie $x_{i_{1}} \cdots x_{i_{n}}$ is a torsion element of $\mathbb{Q}\left[K_{\Delta}\right]$ as a module over $\mathbb{Q}\left[\mathrm{R}_{1}^{*}\right]$. 


\subsection{An injectivity theorem and freeness}

Suppose that a subtorus $\mathrm{G}$ of $\mathrm{T}$ acts on $\mathcal{Z}_{K}$ locally freely and consider a torus $\mathrm{W}$ such that $\mathrm{G} \subset \mathrm{W} \subset \mathrm{T}$ and $m^{\prime}:=\operatorname{dim} \mathrm{W}$. Let $F \subset \mathcal{Z}_{K}$ be the set of $(m-n)$-dimensional $\mathrm{W}$-orbits, where $\operatorname{dim} \mathrm{G}=m-n$. We have the following injectivity result for the $\mathrm{W}$-action on $\mathcal{Z}_{K}$ when $\left[\mathcal{Z}_{K} / \mathrm{G}\right]$ is a symplectic compact toric orbifold.

Theorem 5.14 Suppose that $\left[\mathcal{Z}_{K} / \mathrm{G}\right]$ is a symplectic compact toric orbifold corresponding to a labeled polytope $(\Delta, b)$. Suppose that the stabilizer of any point $x \in \mathcal{Z}_{K}$ in $\mathrm{W}$ is connected. Then the pullback $H_{\mathrm{W}}^{*}\left(\mathcal{Z}_{K} ; \mathbb{Z}\right) \rightarrow H_{\mathrm{W}}^{*}(F ; \mathbb{Z})$ is injective. In particular, $H_{\mathrm{T}}^{*}\left(\mathcal{Z}_{K} ; \mathbb{Z}\right)$ is free over $\mathbb{Z}\left[(\mathrm{T} / \mathrm{W})^{*}\right]$.

Proof Let $\left\{H_{1}, \ldots, H_{m}\right\}$ be the set of all facets of $\Delta$. The simplicial complex $K$ is the one associated to $\Delta$ and $\tau \in K$ if and only if $\Delta_{\tau}:=\cap_{i \in \tau} H_{i} \neq \varnothing$. Let $F_{a}$ be a connected component of $F$. Then by Holm and the second author [18, Lemma 3.4] and our assumption of connected isotropy groups, the stabilizers of every $x \in F_{a}$ in $W$ coincide. Let $W_{a}$ be the stabilizer of points in $F_{a}$. Let $\mu: \mathcal{Z}_{K} \rightarrow \Delta$ be the moment map. Note that this is the quotient map by the action of T. First we show the following lemma.

Lemma 5.15 $F_{a}=\mu^{-1}\left(\Delta_{\sigma}\right)$ for some $\sigma \in K$. In particular, the stabilizer of each point $x \in F_{a}$ is $\mathrm{W}_{\sigma}:=\mathrm{W} \cap \mathrm{T}_{\sigma}$.

Proof of Lemma 5.15 For $x \in F_{a}$, let $\sigma_{x}:=\left\{i \in[m], x_{i}=0\right\}$. Then $\mu^{-1}\left(\Delta_{\sigma_{x}}\right) \subset F_{a}$ and the unique stabilizer for $F_{a}$ is given by $\mathrm{W}_{a}=\mathrm{W} \cap \mathrm{T}_{\sigma_{x}}$. Note that $\sigma_{x} \neq \varnothing$ since $m>n$. It suffices to show that there is an element $x \in F_{a}$ such that $\sigma_{x}$ is the unique minimal subset among the collection of subsets, $\left\{\sigma_{y} \mid y \in F_{a}\right\}$. Let $\sigma_{x}$ and $\sigma_{y}$ be minimal for some $x, y \in F_{a}$. Suppose that $\sigma_{x} \neq \sigma_{y}$ and consider $z \in \mu^{-1}\left(\Delta_{\sigma_{x} \cap \sigma_{y}}\right)$ such that $\sigma_{z}=\sigma_{x} \cap \sigma_{y}$. Since $W_{a}=\mathrm{W} \cap \mathrm{T}_{x} \cap \mathrm{T}_{\sigma_{y}}=\mathrm{W}_{z}, z \in F$ by dimension counting. The connectivity of $F_{a}$ then implies that $z \in F_{a}$. This contradicts the assumption that $\sigma_{x}$ and $\sigma_{y}$ are minimal, so $\sigma_{x}=\sigma_{y}$.

Now let $\left\{F_{\sigma}\right\}$ be the set of connected components of $F$ where $F_{\sigma}=\mu^{-1}\left(\Delta_{\sigma}\right)$. For each $\sigma$, choose a splitting $\mathrm{W}=\mathrm{W}_{\sigma} \times\left(\mathrm{W} / \mathrm{W}_{\sigma}\right)$. The target of the injectivity map is computed as follows:

$$
\begin{aligned}
H_{\mathrm{W}}^{*}(F ; \mathbb{Z}) & =\bigoplus_{\sigma} H_{\mathrm{W}}^{*}\left(F_{\sigma} ; \mathbb{Z}\right)=\bigoplus_{\sigma} H^{*}\left(B \mathrm{~W} \times_{\mathrm{W}} F_{\sigma} ; \mathbb{Z}\right) \\
& =\bigoplus_{\sigma} H^{*}\left(B W_{\sigma} ; \mathbb{Z}\right) \otimes H^{*}\left(F_{\sigma} /\left(\mathrm{W} / \mathrm{W}_{\sigma}\right) ; \mathbb{Z}\right)
\end{aligned}
$$


Now we show that $F_{\sigma} /\left(\mathrm{W} / \mathrm{W}_{\sigma}\right)$ is a compact toric symplectic manifold so that $H^{*}\left(F_{\sigma} /\left(\mathrm{W} / \mathrm{W}_{\sigma}\right) ; \mathbb{Z}\right)$ has only even degree and has no $\mathbb{Z}$-torison. Since $\left[F_{\sigma} / \mathrm{G}\right]$ is the suborbifold of $(\mathrm{W} / \mathrm{G})$-fixed orbifold points, it is a symplectic orbifold (cf [21, page 4210, Corollary 3.8]), which is compact. Since the unique stabilizer of points of $F_{\sigma}$ in $\mathrm{G}$ is given by $\mathrm{G}_{\sigma}=\mathrm{G} \cap \mathrm{W}_{\sigma}$, the quotient $F_{\sigma} /\left(\mathrm{G} / \mathrm{G}_{\sigma}\right)$ is a compact toric manifold with the effective Hamiltonian action of $(\mathrm{T} / \mathrm{G}) /\left(\mathrm{T}_{\sigma} / \mathrm{G}_{\sigma}\right)$. On the other hand, $F_{\sigma} /\left(\mathrm{G} / \mathrm{G}_{\sigma}\right)$ is exactly $F_{\sigma} / \mathrm{W}=F_{\sigma} /\left(\mathrm{W} / \mathrm{W}_{\sigma}\right)$. Thus $H^{*}\left(F_{\sigma} /\left(\mathrm{W} / \mathrm{W}_{\sigma}\right) ; \mathbb{Z}\right)$ has only even degree and has no $\mathbb{Z}$-torison. Therefore, if we show the injectivity of $H_{\mathrm{W}}^{*}\left(\mathcal{Z}_{K} ; \mathbb{Z}\right) \rightarrow H_{\mathrm{W}}^{*}(F ; \mathbb{Z})$, it follows that $H_{\mathrm{W}}^{*}\left(\mathcal{Z}_{K} ; \mathbb{Z}\right)$ has no $\mathbb{Z}$-torison and has only even degree. Then we also have freeness of $H_{\mathrm{T}}^{*}\left(\mathcal{Z}_{K} ; \mathbb{Z}\right)$ over $\mathbb{Z}\left[(\mathrm{T} / \mathrm{W})^{*}\right]$ by Proposition 5.5.

To show injectivity of $H_{\mathrm{W}}^{*}\left(\mathcal{Z}_{K} ; \mathbb{Z}\right) \rightarrow H_{\mathrm{W}}^{*}(F ; \mathbb{Z})$, we apply [18, Theorem 4.10]. It suffices to show that $W_{\sigma}$ is connected and the weights of the action on the (negative) normal bundle are all primitive for each connected component of $F_{\sigma}$. The former is true by the assumption. For the latter, look at the normal bundle of $F_{\sigma}$ in $\mathcal{Z}_{K}$ which is given by $\bigoplus_{i \in \sigma} \mathbb{C}\left(\partial / \partial z_{i}\right)$. The weights of the $\mathrm{T}_{\sigma}$-equivariant normal bundle are the standard $\mathbb{Z}$-basis $\left\{\lambda_{i}, i \in \sigma\right\}$ of $\mathrm{N}_{\mathrm{T}_{\sigma}}^{*}$. We need to check that the induced $W_{\sigma}$-weights $\tilde{\lambda}_{i}:=A_{\sigma}^{*}\left(\lambda_{i}\right) \in \mathrm{N}_{\mathrm{W}_{\sigma}}^{*}$ are nonzero and primitive where $A_{\sigma}: W_{\sigma} \hookrightarrow T_{\sigma}$ is the restriction of the natural inclusion $A: \mathrm{W} \rightarrow \mathrm{T}$. It is easy to see that $\tilde{\lambda}_{i}$ is nonzero, since, if otherwise, the normal direction $\mathbb{C}\left(\partial / \partial z_{i}\right)$ is also contained in $F_{a}$. Finally the proof is completed by the following lemma.

Lemma 5.16 $A_{\sigma}^{*}\left(\lambda_{i}\right) \in \mathrm{N}_{\mathrm{W}_{\sigma}}^{*}$ is primitive.

Proof of Lemma 5.16 Consider the following commutative diagram of tori and its dual for the weight lattices:
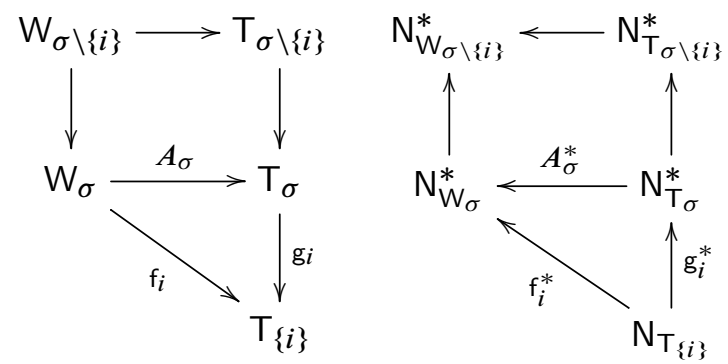

Here $\mathrm{g}_{i}$ is the canonical projection. The map $\mathrm{f}_{i}=\mathrm{g}_{i} \circ A_{\sigma}$ must be surjective since $\mathrm{T}_{i}$ is one-dimensional and $\tilde{\lambda}_{i}$ is nonzero. Also we have $W_{\sigma \backslash\{i\}}=\operatorname{ker}_{i}$ which must be connected by assumption. Therefore we have a short exact sequence of tori $0 \rightarrow \mathrm{W}_{\sigma \backslash\{i\}} \rightarrow \mathrm{W}_{\sigma} \rightarrow \mathrm{T}_{\{i\}} \rightarrow 0$ which implies that $f_{i}^{*}$ maps $N_{\mathrm{T}_{i}}^{*}$ to a direct summand. Thus $\tilde{\lambda}_{i}$ must be primitive since $\lambda_{i}$ is a basis of $\mathrm{N}_{\mathrm{T}_{i}}^{*}$. 
This completes the proof of Theorem 5.14.

Remark 5.17 To give some ideas of how $W$ can be chosen, please see Example 6.1. In the extreme case when $\mathrm{W}=\mathrm{G}$, the assumption implies that $F=\mathcal{Z}_{K}$ and $\mathrm{G}$ acts on $\mathcal{Z}_{K}$ freely. Thus Theorem 5.14 is nothing but the fact that the equivariant cohomology of toric manifolds (or smooth toric varieties) is free over $\mathbb{Z}\left[\mathrm{R}^{*}\right]$.

\section{Examples}

Example 6.1 (Effective weighted projective spaces) Let a $:=\left(a_{1}, \ldots, a_{m}\right)$ be a sequence of positive integers with $\operatorname{gcd}\left(a_{1}, \ldots, a_{m}\right)=1$ and let $\left[\mathbb{P}_{a}^{m-1}\right]$ be the corresponding effective weighed projective space. As in Section $2, H^{*}\left(\left[\mathbb{C P}_{a}^{m-1}\right] ; \mathbb{Z}\right)=$ $H_{\mathrm{G}}^{*}\left(\mathcal{Z}_{K} ; \mathbb{Z}\right)$, where $\mathrm{G}=\left\{\left(t^{a_{1}}, \ldots, t^{a_{m}}\right)\right\} \subset \mathrm{T}$ and $K$ the boundary of an $(m-1)-$ simplex. The corresponding Stanley-Reisner ring is $\mathbb{Z}\left[x_{1}, \ldots, x_{m}\right] /\left\langle x_{1} x_{3} \cdots x_{m}\right\rangle$. In [17], the ordinary cohomology is computed as $H_{\mathrm{G}}^{*}\left(\mathcal{Z}_{K}, \mathbb{Z}\right) \cong \mathbb{Z}[y] /\left\langle a_{1} \cdots a_{m} y^{m}\right\rangle$. It has only even degree, so $H_{\mathrm{T}}^{*}\left(\mathcal{Z}_{K} ; \mathbb{Z}\right) \rightarrow H_{\mathrm{G}}^{*}\left(\mathcal{Z}_{K} ; \mathbb{Z}\right)$ is surjective. However $H_{\mathrm{G}}^{*}\left(\mathcal{Z}_{K} ; \mathbb{Z}\right)$ has $\mathbb{Z}$-torison, and so $H_{\mathrm{T}}^{*}\left(\mathcal{Z}_{K}, \mathbb{Z}\right)$ is not free over $\mathbb{Z}\left[\mathrm{R}^{*}\right]$. The simplest such example is $\left[\mathbb{C P}_{12}^{1}\right]$. In light of Theorem 5.14 , we can find a subtorus $\mathrm{W} \subset \mathrm{T}$ such that $\mathrm{G} \subset \mathrm{W}$ and $\mathbb{Z}[\mathrm{K}]$ is free over $\mathbb{Z}\left[(\mathrm{T} / \mathrm{W})^{*}\right]$ as follows. Define $\mathrm{W} \subset \mathrm{T}$ by $\mathrm{W}=$ $\left\{\left(t_{1}, \ldots, t_{m}\right) \in \mathrm{T} \mid t_{i}=t_{j}\right.$ if $\left.a_{i}=a_{j}\right\}$. Such $\mathrm{W}$ contains $\mathrm{G}$ and the stabilizer of any point $x \in \mathcal{Z}_{K}$ in $\mathrm{W}$ is connected. Therefore by Theorem $5.14, \mathbb{Z}[\mathrm{K}]$ is free over $\mathbb{Z}\left[(\mathrm{T} / \mathrm{W})^{*}\right]$.

Example 6.2 As we saw in the previous examples, $H_{\mathrm{G}}^{*}\left(\mathcal{Z}_{K} ; \mathbb{Z}\right)$ has $\mathbb{Z}$-torison (infinitely many) in even degree for $\left[\mathcal{Z}_{K} / G\right]=\mathbb{C P}_{a}^{m-1}$. The direct product of such toric orbifolds is also a toric orbifold and the Künneth theorem provides the $\mathbb{Z}$-torisons in odd degree. More concretely, take the labeled polytope

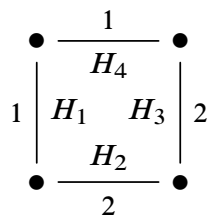

which gives $B=\left(\begin{array}{cccc}1 & 0 & -2 & 0 \\ 0 & 2 & 0 & -1\end{array}\right)$. This defines the direct product $\left[\mathbb{C} \mathbb{P}_{12}^{1} \times \mathbb{C P}_{12}^{1}\right]$ which gives odd degree elements in $H_{\mathrm{T}}^{*}\left(\mathcal{Z}_{K}, \mathbb{Z}\right)$. Thus $H_{\mathrm{T}}^{*}\left(\mathcal{Z}_{K}, \mathbb{Z}\right) \rightarrow H_{\mathrm{G}}^{*}\left(\mathcal{Z}_{K}, \mathbb{Z}\right)$ is not surjective. We can also see this by determining whether $\left(x_{1}-2 x_{3}, 2 x_{2}-x_{4}\right)$ is a regular sequence of $\mathbb{Z}[K]=\mathbb{Z}\left[x_{1}, \ldots, x_{4}\right] /\left\langle x_{1} x_{3}, x_{2} x_{4}\right\rangle$ as a module over $\mathbb{Z}\left[x_{1}-2 x_{3}, 2 x_{2}-x_{4}\right]$. Indeed, it is not a regular sequence: $x_{1}-2 x_{3}$ is a nonzero divisor in $\mathbb{Z}[K]$ but $2 x_{2}-x_{4}$ is a zero divisor in $\mathbb{Z}[K] /\left(x_{1}-2 x_{3}\right)$ since $\left(2 x_{2}-x_{4}\right) x_{2} x_{3}^{2}=0$ and $x_{2} x_{3}^{2} \neq 0$ in $\mathbb{Z}[K] /\left(x_{1}-2 x_{3}\right)$. 


\section{Algebraic Gysin sequence}

Let $\mathrm{U}$ be a subgroup of $\mathrm{G}$ such that $\mathrm{L}:=\mathrm{G} / \mathrm{U}$ is a 1 -dimensional torus. By choosing an identification of $L$ with a standard circle $U(1)$, we have an oriented principal $\mathrm{U}(1)$-bundle $\pi: E \mathrm{~T} \times_{\mathrm{U}} \mathcal{Z}_{K} \rightarrow E \mathrm{~T} \times_{\mathrm{G}} \mathcal{Z}_{K}$ and the corresponding Gysin sequence

$$
\begin{array}{r}
\cdots \longrightarrow H_{\mathrm{G}}^{i-1}\left(\mathcal{Z}_{K}, \mathbb{Z}\right) \\
\stackrel{\cup e}{\longrightarrow} H_{\mathrm{G}}^{i+1}\left(\mathcal{Z}_{K}, \mathbb{Z}\right) \stackrel{\pi^{*}}{\longrightarrow} H_{\mathrm{U}}^{i+1}\left(\mathcal{Z}_{K}, \mathbb{Z}\right) \stackrel{\pi_{*}}{\longrightarrow} H_{\mathrm{G}}^{i}\left(\mathcal{Z}_{K}, \mathbb{Z}\right) \\
\stackrel{\cup e}{\longrightarrow} H_{\mathrm{G}}^{i+2}\left(\mathcal{Z}_{K}, \mathbb{Z}\right) \longrightarrow \cdots
\end{array}
$$

where $\boldsymbol{e}$ is the Euler class of the bundle, $\pi_{*}$ is the push forward map and $\pi^{*}$ is the pullback map. In light of Theorem 3.3, it is natural to ask if there is a purely algebraic construction of a long exact sequence of Tor's corresponding the Gysin sequence. It turns out that it comes from the long exact sequence associated to the iterative construction of the commutative Koszul complex. We describe the construction in the following.

Construction 7.1 (Algebraic Gysin sequence) Let $\widetilde{R}:=T / U$ and make the identification $H^{*}(B \widetilde{\mathrm{R}}, \mathbb{Z})=\mathbb{Z}\left[u_{1}, \ldots, u_{n}, u_{n+1}\right]=\mathbb{Z}\left[\widetilde{\mathrm{R}}^{*}\right]$, where $\mathbb{Z}\left[u_{1}, \ldots, u_{n}\right]=H^{*}(B \mathrm{R}, \mathbb{Z})$. Consider the short exact sequence of Koszul complexes (as modules over $\mathbb{Z}\left[\widetilde{R}^{*}\right]$ )

(7) $0 \rightarrow K^{\mathbb{Z}\left[\widetilde{\mathrm{R}}^{*}\right]}\left(\xi_{1}, \ldots, \xi_{n}\right) \stackrel{\tau^{*}}{\rightarrow} K^{\mathbb{Z}\left[\widetilde{\mathrm{R}}^{*}\right]}\left(\xi_{1}, \ldots, \xi_{n}, \xi_{n+1}\right) \stackrel{\tau_{*}}{\rightarrow} K^{\mathbb{Z}\left[\tilde{\mathrm{R}}^{*}\right]}\left(\xi_{1}, \ldots, \xi_{n}\right) \rightarrow 0$,

where the map $\tau^{*}$ is the obvious inclusion and the map $\tau_{*}$ is getting rid of $\xi_{n+1} \wedge$, lowering the homological degree by 1 . Note that

$$
K^{\mathbb{Z}\left[\tilde{\mathrm{R}}^{*}\right]}\left(\xi_{1}, \ldots, \xi_{n}\right)=\mathbb{Z}\left[u_{1}, \ldots, u_{n}, u_{n+1}\right]\left\langle\xi_{1}, \ldots, \xi_{n}\right\rangle
$$

and the differential is given by extending $\partial \xi_{i}=u_{i}$ as a differential algebra, where \langle\rangle denotes the exterior algebra.

Let $M$ be a graded $\mathbb{Z}\left[x_{1}, \ldots, x_{m}\right]$-module. After tensoring with $M$ over $\mathbb{Z}\left[\widetilde{R}^{*}\right]$, we obtain the long exact sequence of Tor modules over $\mathbb{Z}\left[u_{1}, \ldots, u_{n}, u_{n+1}\right]$ :

$$
\cdots \stackrel{\delta}{\longrightarrow} \operatorname{Tor}_{i+1}^{\mathbb{Z}\left[\mathrm{R}^{*}\right]}(\mathrm{M}, \mathbb{Z}) \stackrel{\tau^{*}}{\longrightarrow} \operatorname{Tor}_{i+1}^{\mathbb{Z}\left[\tilde{\mathrm{R}}^{*}\right]}(\mathrm{M}, \mathbb{Z}) \stackrel{\tau_{*}}{\longrightarrow} \operatorname{Tor}_{i}^{\mathbb{Z}\left[\mathrm{R}^{*}\right]}(\mathrm{M}, \mathbb{Z}) \stackrel{\delta}{\longrightarrow} \cdots
$$

We call this the algebraic (homological) Gysin sequence. 
Proposition 7.2 The connecting map $\delta$ is a multiplication by $u_{n+1}$.

Proof It follows from a diagram chase. Consider the part of the map (7) of complexes:

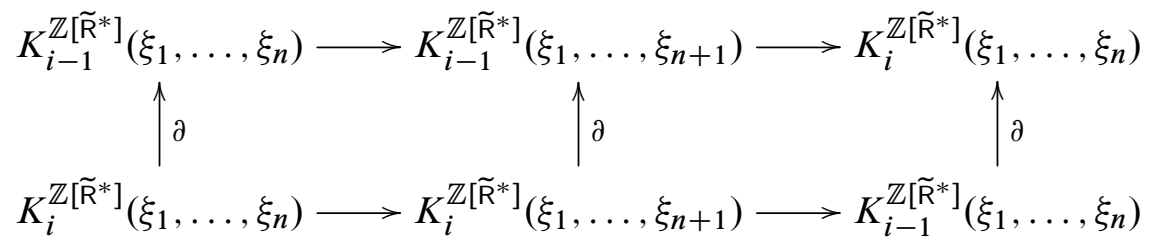

Let $z$ be a cycle in $K_{i-1}^{\mathbb{Z}\left[\tilde{\mathrm{R}}^{*}\right]}\left(\xi_{1}, \ldots, \xi_{n}\right)$ and lift it to top left corner:

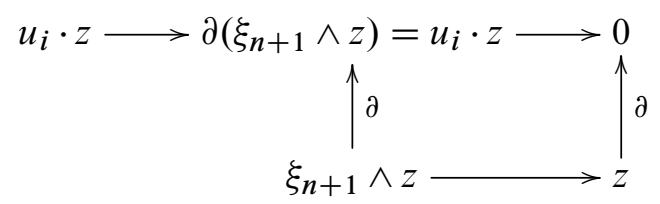

This concludes the proof.

Definition 7.3 (Cohomological algebraic Gysin sequence) As in Definition 2.2, we can assign the cohomological degree and turn the sequence (8) into a cohomological sequence:

$$
\begin{aligned}
\cdots \stackrel{\cdot u_{n+1}}{\longrightarrow} \operatorname{Tor}_{\mathbb{Z}\left[\mathrm{R}^{*}\right]}^{i+1}(\mathrm{M}, \mathbb{Z}) \stackrel{\tau^{*}}{\longrightarrow} \operatorname{Tor}_{\mathbb{Z}\left[\widetilde{\mathrm{R}}^{*}\right]}^{i+1}(\mathrm{M}, \mathbb{Z}) \\
\stackrel{\tau_{*}}{\longrightarrow} \operatorname{Tor}_{\mathbb{Z}\left[\mathrm{R}^{*}\right]}^{i}(\mathrm{M}, \mathbb{Z}) \stackrel{\cdot u_{n+1}}{\longrightarrow} \cdots
\end{aligned}
$$

We call this the cohomological algebraic Gysin sequence.

Remark 7.4 We remark here that the above sequence depends on the choice of $u_{n+1}$, which is unique up to a sign and a choice of an element of $\mathbb{Z}\left[u_{1}, \ldots, u_{n}\right]=\mathbb{Z}\left[\mathrm{R}^{*}\right]$. This is because the choice of $u_{n+1}$ is nothing more than a choice of complement of $\mathrm{R}$ in $\widetilde{R}$. The choice of sign persists to (9), but the element of $\mathbb{Z}\left[R^{*}\right]$ vanishes upon taking cohomology, since elements of $\mathbb{Z}\left[R^{*}\right]$ are boundaries in the image. On the topological side, this choice of sign amounts to the choice of orientation needed to define the Euler class of the U(1)-bundle $\pi$. 
Remark 7.5 In the special case when $K$ is the simplicial complex associated to a Delzant polytope $\Delta$, the corresponding $G$-action on $\mathcal{Z}_{K}$ is free and $\mathcal{Z}_{K} / G$ is homeomorphic to the corresponding symplectic toric manifold. We have

$$
\operatorname{Tor}_{i}^{\mathbb{Z}\left[\mathrm{R}^{*}\right]}(\mathbb{Z}[K], \mathbb{Z})=0 \quad \text { for all } i \geq 1
$$

since $\mathbb{Z}[K]$ is free over $\mathbb{Z}\left[\mathrm{R}^{*}\right]$. Thus the long exact sequence in Construction 7.1 implies that

$$
\operatorname{Tor}_{i}^{\mathbb{Z}\left[\widetilde{R}^{*}\right]}(\mathbb{Z}[K], \mathbb{Z})=0 \quad \text { for all } i \geq 2 .
$$

Hence the only nonzero part of the long exact sequence is:

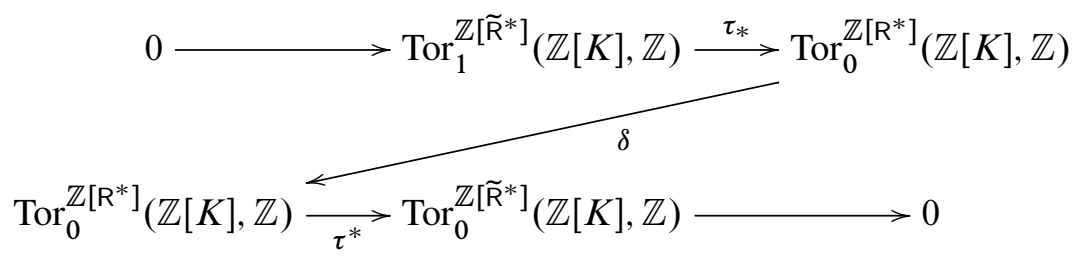

This sequence, together with the identification of Tor algebras and cohomology rings of toric manifolds, gives the Gysin sequence used in the first author's paper [22] to compute the cohomology ring of a good contact toric manifold.

\section{References}

[1] A Adem, J Leida, Y Ruan, Orbifolds and stringy topology, Cambridge Tracts in Mathematics 171, Cambridge Univ. Press (2007) MR2359514

[2] I V Baskakov, V M Bukhshtaber, T E Panov, Algebras of cellular cochains, and torus actions, Uspekhi Mat. Nauk 59 (2004) 159-160 MR2117435

[3] L A Borisov, L Chen, G G Smith, The orbifold Chow ring of toric Deligne-Mumford stacks, J. Amer. Math. Soc. 18 (2005) 193-215 MR2114820

[4] W Bruns, J Herzog, Cohen-Macaulay rings, Cambridge Studies in Advanced Mathematics 39, Cambridge Univ. Press (1993) MR1251956

[5] V M Buchstaber, T E Panov, Torus actions and their applications in topology and combinatorics, University Lecture Series 24, Amer. Math. Soc. (2002) MR1897064

[6] V M Bukhshtaber, T E Panov, Torus actions and the combinatorics of polytopes, Tr. Mat. Inst. Steklova 225 (1999) 96-131 MR1725935

[7] D A Cox, The homogeneous coordinate ring of a toric variety, J. Algebraic Geom. 4 (1995) 17-50 MR1299003

[8] V I Danilov, The geometry of toric varieties, Uspekhi Mat. Nauk 33 (1978) 85-134, 247 MR495499 
[9] M W Davis, T Januszkiewicz, Convex polytopes, Coxeter orbifolds and torus actions, Duke Math. J. 62 (1991) 417-451 MR1104531

[10] D Edidin, Equivariant geometry and the cohomology of the moduli space of curves arXiv:1006. 2364

[11] D Edidin, W Graham, Equivariant intersection theory, Invent. Math. 131 (1998) 595-634 MR1614555

[12] M Franz, Koszul duality and equivariant cohomology for tori, Int. Math. Res. Not. 2003 (2003) 2255-2303 MR1997219

[13] M Franz, The integral cohomology of toric manifolds, Tr. Mat. Inst. Steklova 252 (2006) 61-70 MR2255969

[14] M Franz, Describing toric varieties and their equivariant cohomology, Colloq. Math. 121 (2010) 1-16 MR2725697

[15] M Franz, V Puppe, Exact cohomology sequences with integral coefficients for torus actions, Transform. Groups 12 (2007) 65-76 MR2308029

[16] A Hatcher, Algebraic topology, Cambridge Univ. Press (2002) MR1867354

[17] TS Holm, Orbifold cohomology of abelian symplectic reductions and the case of weighted projective spaces, from: "Poisson geometry in mathematics and physics", (G Dito, J-H Lu, Y Maeda, A Weinstein, editors), Contemp. Math. 450, Amer. Math. Soc. (2008) 127-146 MR2397623

[18] T S Holm, T Matsumura, Equivariant cohomology for Hamiltonian torus actions on symplectic orbifolds, Transform. Groups 17 (2012) 717-746 MR2956164

[19] J Jurkiewicz, Torus embeddings, polyhedra, $k^{*}$-actions and homology, Dissertationes Math. (Rozprawy Mat.) 236 (1985) 64 MR820078

[20] E Lerman, A Malkin, Hamiltonian group actions on symplectic Deligne-Mumford stacks and toric orbifolds, Adv. Math. 229 (2012) 984-1000 MR2855084

[21] E Lerman, S Tolman, Hamiltonian torus actions on symplectic orbifolds and toric varieties, Trans. Amer. Math. Soc. 349 (1997) 4201-4230 MR1401525

[22] S Luo, Cohomology rings of good contact toric manifolds arXiv:1012.2146

[23] H Matsumura, Commutative ring theory, 2nd edition, Cambridge Studies in Advanced Mathematics 8, Cambridge Univ. Press (1989) MR1011461

[24] J McCleary, A user's guide to spectral sequences, 2nd edition, Cambridge Studies in Advanced Mathematics 58, Cambridge Univ. Press (2001) MR1793722

[25] M Poddar, S Sarkar, On quasitoric orbifolds, Osaka J. Math. 47 (2010) 1055-1076 MR2791564

[26] M Romagny, Group actions on stacks and applications, Michigan Math. J. 53 (2005) 209-236 MR2125542 
[27] J-P Serre, Local algebra, Springer Monographs in Mathematics, Springer, Berlin (2000) MR1771925

[28] S Tolman, Group actions and cohomology, $\mathrm{PhD}$ thesis, Harvard University, Ann Arbor, MI (1993) MR2689600 Available at http://search.proquest.com/docview/ 304085837

Department of Mathematics, Cornell University 310 Malott Hall, Ithaca, NY 14853, USA

Department of Mathematical Sciences, KAIST

291 Daehak-ro, Yuseong-gu Daejeon, Daejeon 305-701, South Korea

Department of Mathematics, Wake Forest University

PO Box 7388, 127 Manchester Hall, Winston-Salem, NC 27109, USA

sl943@cornell.edu, tomoomatsumura@kaist.ac.kr, moorewf@wfu.edu

Received: 5 August 2012 Revised: 10 March 2013 